\title{
Facile Synthesis of the IndenoTetrahydropyridine Core of Haouamine A
}

\author{
Nicole D.Smith, Joji Hayashida, and Viresh H. Rawal \\ Department of Chemistry, The University of Chicago \\ 5735 South Ellis Avenue, Chicago, IL 60637
}

\section{SUPPORTING INFORMATION}

\section{General}

All reagents were commercially obtained (Aldrich, Acros) at the highest commercial quality and used without further purification. Air and moisture sensitive reagents were transferred via syringe or cannula. All moisture sensitive reactions were carried out in oven-dried glassware under an atmosphere of argon with freshly distilled solvents. The reactions were monitored by thin-layer chromatography carried out on $0.25 \mathrm{~mm}$ Whatman silica gel plates using UV light as the visualizing agent. The NMR spectra were obtained on a Bruker DRX-400 (400 MHz) or a Bruker DRX-500 (500 MHz) spectrometer. Chemical shifts $(\delta)$ are reported in parts per million $(\mathrm{ppm})$ relative to deuterated solvent signals in $\mathrm{d}_{6}$-DMSO. The following abbreviations were used to explain the multiplicities: $\mathrm{s}=$ singlet; $\mathrm{d}=$ doublet, $\mathrm{t}=$ triplet, $\mathrm{q}=$ quartet, $\mathrm{dd}=\mathrm{doublet}$ of doublets, $m=$ multiplet, $b=$ broad. Mass spectroscopic analyses were carried out at the facilities of The University of Chicago or The Ohio State University. IR spectra were recorded on a Nicolet Nexus 670 FT-IR and values are reported in $\mathrm{cm}^{-1}$ units.

\section{3-Oxo-piperidine-1-carboxylic acid methyl ester (6)}

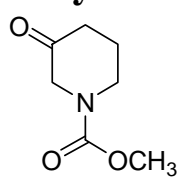

To a solution of 3-hydroxypiperidine hydrochloride $(4.82 \mathrm{~g}, 35.0 \mathrm{mmol})$ in anhydrous $\mathrm{CH}_{2} \mathrm{Cl}_{2}(100 \mathrm{~mL})$ was added TEA $(9.76 \mathrm{~mL}, 70.0 \mathrm{mmol})$ and the mixture was cooled to $0{ }^{\circ} \mathrm{C}$ in an ice bath. A solution of dimethyl dicarbonate $(4.12 \mathrm{~mL}, 38.5 \mathrm{mmol})$ in anhydrous $\mathrm{CH}_{2} \mathrm{Cl}_{2}(10 \mathrm{~mL})$ was then added dropwise via addition funnel, and the reaction mixture was allowed to warm to room temperature and stir overnight. The $\mathrm{CH}_{2} \mathrm{Cl}_{2}$ was removed in vacuo, and $\mathrm{Et}_{2} \mathrm{O}$ was added to precipitate out the ammonium salts. The precipitate was filtered and washed with $\mathrm{Et}_{2} \mathrm{O}(3 \mathrm{X}, 20 \mathrm{~mL})$. The crude product was purified by flash silica gel chromatography $\left(9: 1, \mathrm{Et}_{2} \mathrm{O}: \mathrm{Hex}\right)$ to afford 3-hydroxypiperidine-1-carboxylic acid methyl ester $(5.57 \mathrm{~g}, 35.0 \mathrm{mmol})$ in quantitative yield. 
Anhydrous $\mathrm{CH}_{2} \mathrm{Cl}_{2}(240 \mathrm{~mL})$ was added to a $500 \mathrm{~mL}$ round bottom flask, purged with $\mathrm{N}_{2}$ and cooled to $-78^{\circ} \mathrm{C}$. Oxalyl chloride $(3.45 \mathrm{~mL}, 39.5 \mathrm{mmol})$ was added, followed by the dropwise addition of anhydrous DMSO $(4.69 \mathrm{~mL}, 66.0 \mathrm{mmol})$. This solution was allowed to stir at $-78{ }^{\circ} \mathrm{C}$ for $5 \mathrm{~min}$. Next, 3-hydroxy-piperidine-1-carboxylic acid methyl ester (5.25 g, $33.0 \mathrm{mmol})$ dissolved in anhydrous $\mathrm{CH}_{2} \mathrm{Cl}_{2}(100 \mathrm{~mL})$ was added dropwise via cannula to the reaction mixture at $-78^{\circ} \mathrm{C}$. After $30 \mathrm{~min}$, TEA $(18.4 \mathrm{~mL}, 132 \mathrm{mmol})$ was added and the reaction mixture was allowed to warm to $\mathrm{rt}$ and stir for $2 \mathrm{~h}$. The reaction mixture was diluted with $\mathrm{CH}_{2} \mathrm{Cl}_{2}(100 \mathrm{~mL})$, washed with sat. $\mathrm{NH}_{4} \mathrm{Cl}(150 \mathrm{~mL})$, sat. $\mathrm{NaHCO}_{3}(150 \mathrm{~mL})$, brine $(150 \mathrm{~mL})$ and dried with $\mathrm{MgSO}_{4}$. The organic layer was concentrated in vacuo and purified via flash silica gel chromatography (3:2, EtOAc:Hex) to afford 3-oxo-piperidine-1-carboxylic acid methyl ester $(4.98 \mathrm{~g}, 31.7 \mathrm{mmol})$ in a 96\% yield. ${ }^{1} \mathrm{H}$ NMR $\left(\mathrm{d}_{6}\right.$-DMSO, $\left.400 \mathrm{MHz}, 363 \mathrm{~K}\right) \delta 1.61-1.68(\mathrm{~m}, 2 \mathrm{H}), 2.14(\mathrm{t}, 2 \mathrm{H}, \mathrm{J}=7.0 \mathrm{~Hz})$, 3.27 (t, 2H, J=6.0 Hz), 3.35 (s, 3H), 3.66 (s, 2H); ${ }^{13} \mathrm{C}$ NMR (d 6 -DMSO, $\left.400 \mathrm{MHz}, 363 \mathrm{~K}\right)$ $\delta 21.4,37.1,41.5,51.7,53.2,154.7,204.4$; IR $(\mathrm{NaCl})$ v 1226, 1407, 1451, 1700, 2873 , 2956; MS (ESI) $158\left(\mathrm{M}+\mathrm{H}^{+}\right)$.

\section{2-(3-Methoxy-benzyl)-3-oxo-piperidine-1-carboxylic acid methyl ester (7)}

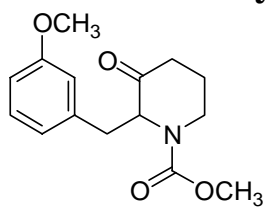

To a solution of 3-oxo-piperidine-1-carboxylic acid methyl ester (2.00 g, $12.7 \mathrm{mmol})$ in anhydrous benzene $(24.0 \mathrm{~mL})$ was added pyrrolidine $(1.57 \mathrm{~mL}, 19.1 \mathrm{mmol})$, and the reaction was allowed to stir for $30 \mathrm{~min}$. Next, the mixture was heated to $110{ }^{\circ} \mathrm{C}$ and $\mathrm{H}_{2} \mathrm{O}$ and benzene were collected utilizing a Dean-Stark trap. The remaining benzene was removed in vacuo and the enamine was redissolved in $\mathrm{CH}_{3} \mathrm{CN}(24.0 \mathrm{~mL})$. To this solution was added a solution of 3-methoxybenzyl chloride $(2.4 \mathrm{~mL}, 16.5 \mathrm{mmol})$ in $\mathrm{CH}_{3} \mathrm{CN}(10 \mathrm{~mL})$, and the reaction mixture was refluxed overnight. The reaction was quenched with brine $(30 \mathrm{~mL})$ and extracted with EtOAc $(2 \mathrm{X}, 50 \mathrm{~mL})$. The organic layer was washed with sat. $\mathrm{NH}_{4} \mathrm{Cl}(40 \mathrm{~mL})$, dried $\left(\mathrm{MgSO}_{4}\right)$, concentrated in vacuo and purified via flash silica gel chromatography (3:2, Hex:EtOAc) to afford 2-(3-methoxy-benzyl)-3oxo-piperidine-1-carboxylic acid methyl ester $(2.03 \mathrm{~g}, 7.32 \mathrm{mmol})$ in a $58 \%$ yield. ${ }^{1} \mathrm{H}$ NMR (d ${ }_{6}$-DMSO, $\left.400 \mathrm{MHz}, 363 \mathrm{~K}\right) \delta 1.83-1.90(\mathrm{~m}, 2 \mathrm{H}), 2.35-2.42(\mathrm{~m}, 1 \mathrm{H}), 2.49-2.59(\mathrm{~m}$, $1 \mathrm{H}), 2.97-3.02(\mathrm{~m}, 2 \mathrm{H}), 3.18-3.25(\mathrm{~m}, 1 \mathrm{H}), 3.47(\mathrm{~s}, 3 \mathrm{H}), 3.74(\mathrm{~s}, 3 \mathrm{H}), 3.85-3.91(\mathrm{~m}, 1 \mathrm{H})$, $4.59(\mathrm{t}, 1 \mathrm{H}, \mathrm{J}=7.0 \mathrm{~Hz}), 6.73-6.79(\mathrm{~m}, 3 \mathrm{H}), 7.17(\mathrm{t}, 1 \mathrm{H}, \mathrm{J}=8.0 \mathrm{~Hz}) ;{ }^{13} \mathrm{C}$ NMR $\left(\mathrm{d}_{6}-\mathrm{DMSO}\right.$, $400 \mathrm{MHz}, 363 \mathrm{~K}) \delta 21.8,35.8,35.9,38.5,51.5,54.5,63.8,111.7,114.5,120.9,128.5$,

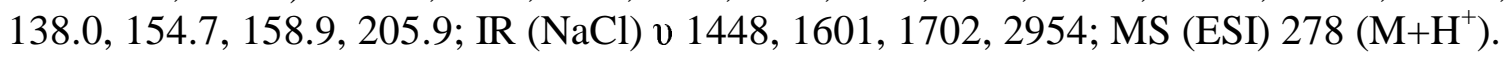




\section{2-(3-Methoxy-benzyl)-3-oxo-3,6-dihydro-2H-pyridine-1-carboxylic acid methyl ester}

(8)

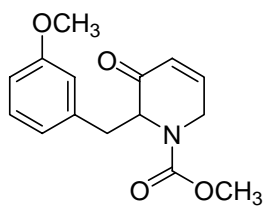

To a solution of 2-(3-methoxy-benzyl)-3-oxo-piperidine-1-carboxylic acid methyl ester $(0.30 \mathrm{~g}, 1.08 \mathrm{mmol})$ in anhydrous DMSO $(3 \mathrm{~mL})$ was added IBX $(0.30 \mathrm{~g}, 1.08 \mathrm{mmol})$, and the mixture was heated to $70{ }^{\circ} \mathrm{C}$. The reaction was allowed to stir at $70{ }^{\circ} \mathrm{C}$ for $4 \mathrm{~h}$ then an additional equivalent of IBX was added, and after $4 \mathrm{~h}$, a third equivalent of IBX was added, and the reaction mixture was allowed to stir at $70{ }^{\circ} \mathrm{C}$ overnight. The mixture was filtered and the filtrate was washed with ether $(3 \mathrm{X}, 5 \mathrm{~mL})$. The organic extracts were combined and washed with sat. $\mathrm{NaHCO}_{3}(5 \mathrm{~mL})$, brine $(5 \mathrm{~mL})$, dried with $\mathrm{MgSO}_{4}$, filtered and concentrated to afford 2-(3-methoxy-benzyl)-3-oxo-3,6-dihydro-2H-pyridine1-carboxylic acid methyl ester $(0.27 \mathrm{~g}, 0.98 \mathrm{mmol})$ in a $90 \%$ yield. ${ }^{1} \mathrm{H}$ NMR (d $\mathrm{d}_{6}$-DMSO, $400 \mathrm{MHz}, 363 \mathrm{~K}) \delta 2.89(\mathrm{dd}, 2 \mathrm{H}, \mathrm{J}=6.8 \mathrm{~Hz}, \mathrm{~J}=6.8 \mathrm{~Hz}$ ), $3.46(\mathrm{bs}, 3 \mathrm{H}), 3.75(\mathrm{~s}, 3 \mathrm{H}), 3.93$ (ddd, $1 \mathrm{H}, \mathrm{J}=2.5 \mathrm{~Hz}, \mathrm{~J}=2.5 \mathrm{~Hz}, \mathrm{~J}=21.0 \mathrm{~Hz}$ ), 4.52 (ddd, $1 \mathrm{H}, \mathrm{J}=2.0 \mathrm{~Hz}, \mathrm{~J}=5.0 \mathrm{~Hz}, \mathrm{~J}=21.0 \mathrm{~Hz}$ ), $4.70(\mathrm{dd}, 1 \mathrm{H}, \mathrm{J}=6.0 \mathrm{~Hz}, \mathrm{~J}=8.5 \mathrm{~Hz}), 6.06(\mathrm{dt}, 1 \mathrm{H}, \mathrm{J}=2.5 \mathrm{~Hz}, \mathrm{~J}=10.5 \mathrm{~Hz}), 6.74-6.80(\mathrm{~m}, 3 \mathrm{H})$, 7.18-7.13 (m, 1H), $7.18(\mathrm{t}, 1 \mathrm{H}, \mathrm{J}=8.0 \mathrm{~Hz}) ;{ }^{13} \mathrm{C}$ NMR $\left(\mathrm{d}_{6}-\mathrm{DMSO}, 400 \mathrm{MHz}, 363 \mathrm{~K}\right) \delta 35.4$, 51.6, 54.4, 60.8, 86.9, 111.7, 114.3, 120.8, 124.4, 128.4, 137.6, 146.7, 154.3, 158.7, 193.4; IR (NaCl) v 1449, 1684, 1700, 2954; MS (ESI) $274\left(\mathrm{M}-\mathrm{H}^{+}\right)$.

\section{3-Hydroxy-2-(3-methoxy-benzyl)-3-(3-methoxy-phenyl)-3,6-dihydro-2H-pyridine-1- carboxylic acid methyl ester (9)}

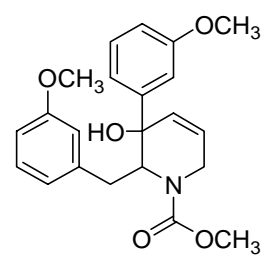

To a solution of 2-(3-methoxy-benzyl)-3-oxo-3,6-dihydro-2H-pyridine-1-carboxylic acid methyl ester $(72.0 \mathrm{mg}, 0.26 \mathrm{mmol})$ in anhydrous THF $(5 \mathrm{~mL})$, was added 3methoxyphenylmagnesium bromide $(523 \mu \mathrm{L}, 0.52 \mathrm{mmol})$ dropwise at rt. The reaction was quenched with $\mathrm{H}_{2} \mathrm{O}(2 \mathrm{~mL})$ after an hour, and extracted with $\mathrm{Et}_{2} \mathrm{O}(3 \mathrm{X}, 5 \mathrm{~mL})$. The organic layers were combined, dried with $\mathrm{MgSO}_{4}$, filtered and concentrated in vacuo. The crude product was purified via flash silica gel chromatography $\left(3: 2, \mathrm{Et}_{2} \mathrm{O}: \mathrm{Hex}\right)$ to afford 3-hydroxy-2-(3-methoxy-benzyl)-3-(3-methoxy-phenyl)-3,6-dihydro-2H-pyridine1-carboxylic acid methyl ester $(75.0 \mathrm{mg}, 0.20 \mathrm{mmol})$ in a $75 \%$ yield. ${ }^{1} \mathrm{H}$ NMR $\left(\mathrm{d}_{6^{-}}\right.$ DMSO, $400 \mathrm{MHz}, 363 \mathrm{~K}) \delta 2.58(\mathrm{dd}, 1 \mathrm{H}, \mathrm{J}=11.0 \mathrm{~Hz}, \mathrm{~J}=14.0 \mathrm{~Hz}$ ), 2.98 (bs, 3H), 3.29 (dd, $1 \mathrm{H}, \mathrm{J}=3.0 \mathrm{~Hz}, \mathrm{~J}=14.5 \mathrm{~Hz}), 3.61-3.65(\mathrm{~m}, 1 \mathrm{H}), 3.73(\mathrm{~s}, 3 \mathrm{H}), 3.74(\mathrm{~s}, 3 \mathrm{H}), 4.17(\mathrm{~m}, 1 \mathrm{H})$, 4.44-4.47 (m, 1H), $5.44(\mathrm{bs}, 1 \mathrm{H}), 5.77-5.79(\mathrm{~m}, 1 \mathrm{H}), 5.99(\mathrm{~d}, 1 \mathrm{H}, \mathrm{J}=10.0 \mathrm{~Hz}), 6.71-6.73$ $(\mathrm{m}, 1 \mathrm{H}), 6.76-6.78(\mathrm{~m}, 2 \mathrm{H}), 6.99-7.00(\mathrm{~m}, 2 \mathrm{H}), 7.11-7.20(\mathrm{~m}, 2 \mathrm{H}) ;{ }^{13} \mathrm{C}$ NMR $\left(\mathrm{d}_{6}-\mathrm{DMSO}\right.$, $400 \mathrm{MHz}, 363 \mathrm{~K}) \delta 33.0,50.9,51.4,54.5,54.5,72.4,80.3,111.1,111.6,111.7,111.7$, 114.2, 117.9, 120.9, 122.7, 127.7, 128.1, 129.6, 140.5, 147.6, 158.4, 158.7; IR (NaCl) v $1455,1600,1680,2923,2953,3426$; HRMS (EM) $[\mathrm{M}+\mathrm{H}]^{+}$calcd for $\mathrm{C}_{22} \mathrm{H}_{25} \mathrm{NO}_{5} \mathrm{Na}$ 406.1624, found 406.1623 . 
5-Methoxy-13-(3-methoxy-phenyl)-10-aza-tricyclo[7.2.2.02,7]trideca-2(7),3,5,12tetraene-10-carboxylic acid methyl ester (10)

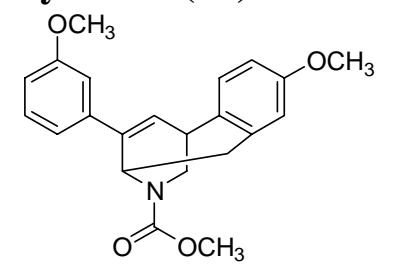

A solution of 3-hydroxy-2-(3-methoxy-benzyl)-3-(3-methoxy-phenyl)-3,6-dihydro-2Hpyridine-1-carboxylic acid methyl ester $(31.0 \mathrm{mg}, 0.08 \mathrm{mmol})$ in anhydrous $\mathrm{CH}_{2} \mathrm{Cl}_{2}(1$ $\mathrm{mL})$ was added dropwise to a $\mathrm{CF}_{3} \mathrm{SO}_{3} \mathrm{H}(1 \mathrm{~mL})$ and $\mathrm{CH}_{2} \mathrm{Cl}_{2}(1 \mathrm{~mL})$ mixture at $-30{ }^{\circ} \mathrm{C}$. The reaction mixture was allowed to stir at $-30{ }^{\circ} \mathrm{C}$ for $30 \mathrm{~min}$ then poured into $\mathrm{H}_{2} \mathrm{O}(10$ $\mathrm{mL}$ ) and extracted with $\mathrm{CH}_{2} \mathrm{Cl}_{2}(3 \mathrm{X}, 25 \mathrm{~mL})$. The organic layers were combined, washed with sat. $\mathrm{NaHCO}_{3}(30 \mathrm{~mL})$, dried with $\mathrm{Na}_{2} \mathrm{SO}_{4}$ and concentrated in vacuo to afford 5methoxy-13-(3-methoxy-phenyl)-10-aza-tricyclo[7.2.2.02,7]trideca-2(7),3,5,12-tetraene10 -carboxylic acid methyl ester $(16.0 \mathrm{mg}, 0.045 \mathrm{mmol})$ in a $55 \%$ yield and 3-methoxy13-(3-methoxy-phenyl)-10-aza-tricyclo[7.2.2.02,7]trideca-2(7),3,5,12-tetraene-10-

carboxylic acid methyl ester $(6.0 \mathrm{mg}, 0.02 \mathrm{mmol})$ in a $21 \%$ yield. ${ }^{1} \mathrm{H}$ NMR $\left(\mathrm{d}_{6}-\mathrm{DMSO}\right.$, $400 \mathrm{MHz}, 363 \mathrm{~K}) \delta 2.98-3.03(\mathrm{~m}, 1 \mathrm{H}), 3.31(\mathrm{dd}, 1 \mathrm{H}, \mathrm{J}=4.5 \mathrm{~Hz}, \mathrm{~J}=18.0 \mathrm{~Hz}), 3.45(\mathrm{dd}, 1 \mathrm{H}$, $\mathrm{J}=4.0 \mathrm{~Hz}, \mathrm{~J}=11.0 \mathrm{~Hz}$ ), 3.61-3.64 (m, 1H), $3.66(\mathrm{bs}, 3 \mathrm{H}), 3.71$, (s, 3H), 3.79 (s, 3H), 5.33 (bs, $1 \mathrm{H}), 6.65-6.68(\mathrm{~m}, 2 \mathrm{H}), 6.87-6.90(\mathrm{~m}, 2 \mathrm{H}), 6.97-6.98(\mathrm{~m}, 1 \mathrm{H}), 7.03(\mathrm{dd}, 1 \mathrm{H}, \mathrm{J}=1.0$ $\mathrm{Hz}, \mathrm{J}=8.0 \mathrm{~Hz}), 7.30(\mathrm{t}, 1 \mathrm{H}, \mathrm{J}=8.0 \mathrm{~Hz}) ;{ }^{13} \mathrm{C}$ NMR $\left(\mathrm{d}_{6}-\mathrm{DMSO}, 400 \mathrm{MHz}, 363 \mathrm{~K}\right) \delta 27.6$, 29.9, 35.9, 50.8, 51.6, 54.6, 54.7, 110.2, 111.1, 112.9, 115.9, 116.7, 128.9, 129.2, 129.6, 131.5, 136.0, 138.4, 154.8, 157.9, 159.3; IR (NaCl) v 1447, 1498, 1605, 1696, 2952; HRMS (EM) $[\mathrm{M}+\mathrm{H}]^{+}$calcd for $\mathrm{C}_{22} \mathrm{H}_{23} \mathrm{NO}_{4} \mathrm{Na} 388.1519$, found 388.1506.

\section{3-Methoxy-13-(3-methoxy-phenyl)-10-aza-tricyclo[7.2.2.02,7]trideca-2(7),3,5,12- tetraene-10-carboxylic acid methyl ester (11)}

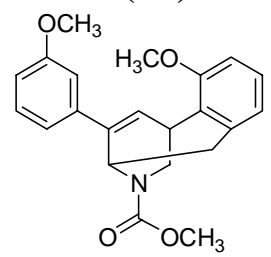

${ }^{1} \mathrm{H}$ NMR $\left(\mathrm{d}_{6}\right.$-DMSO, $\left.400 \mathrm{MHz}, 363 \mathrm{~K}\right) \delta 2.99-3.05(\mathrm{~m}, 1 \mathrm{H}), 3.32(\mathrm{dd}, 1 \mathrm{H}, \mathrm{J}=4.5 \mathrm{~Hz}$, $\mathrm{J}=18.0 \mathrm{~Hz}), 3.47(\mathrm{dd}, 1 \mathrm{H}, \mathrm{J}=4.0 \mathrm{~Hz}, \mathrm{~J}=11.0 \mathrm{~Hz}), 3.62-3.65(\mathrm{~m}, 1 \mathrm{H}), 3.65(\mathrm{bs}, 3 \mathrm{H}), 3.79(\mathrm{~s}$, $3 \mathrm{H}), 3.82(\mathrm{~s}, 3 \mathrm{H}), 4.45-4.47(\mathrm{~m}, 1 \mathrm{H}), 5.32(\mathrm{bs}, 1 \mathrm{H}), 6.68(\mathrm{~d}, 1 \mathrm{H}, \mathrm{J}=8.0 \mathrm{~Hz}), 6.80-6.84(\mathrm{~m}$, $2 \mathrm{H}), 6.88(\mathrm{dd}, 1 \mathrm{H}, \mathrm{J}=2.0 \mathrm{~Hz}, \mathrm{~J}=8.5 \mathrm{~Hz}), 6.98(\mathrm{~m}, 1 \mathrm{H}), 7.03(\mathrm{~d}, 1 \mathrm{H}, \mathrm{J}=8.0 \mathrm{~Hz}), 7.10(\mathrm{t}, 1 \mathrm{H}$, $\mathrm{J}=8.0 \mathrm{~Hz}), 7.30(\mathrm{t}, 1 \mathrm{H}, \mathrm{J}=8.0 \mathrm{~Hz}) ;{ }^{13} \mathrm{C}$ NMR $\left(\mathrm{d}_{6}-\mathrm{DMSO}, 400 \mathrm{MHz}, 363 \mathrm{~K}\right) \delta 20.0,28.4$, $35.8,50.6,51.6,54.7,55.7,108.6,110.1,112.9,116.7,122.9,126.6,127.8,128.9,129.2$, 129.3, 136.3, 138.1, 139.5, 155.1, 159.3; IR (NaCl) v 1447, 1581, 1697, 2923; HRMS (EM) $[\mathrm{M}+\mathrm{H}]^{+}$calcd for $\mathrm{C}_{22} \mathrm{H}_{23} \mathrm{NO}_{4} \mathrm{Na} 388.1519$, found 388.1519 . 
2-(2-Bromo-5-methoxy-benzyl)-3-oxo-piperidine-1-carboxylic acid methyl ester (12)

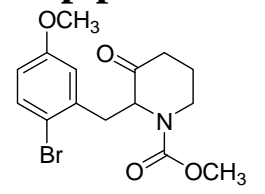

To a solution of 2-(3-methoxy-benzyl)-3-oxo-piperidine-1-carboxylic acid methyl ester $(0.55 \mathrm{~g}, 1.96 \mathrm{mmol})$ in anhydrous $\mathrm{CH}_{3} \mathrm{CN}(5 \mathrm{~mL})$ was added a solution of $\mathrm{N}$ bromosuccinimide $(0.37 \mathrm{~g}, 2.06 \mathrm{mmol})$ in anhydrous $\mathrm{CH}_{3} \mathrm{CN}(5 \mathrm{~mL})$ dropwise via syringe pump. The reaction mixture was allowed to stir for 2 hours, and the $\mathrm{CH}_{3} \mathrm{CN}$ was concentrated in vacuo and the crude product was redissolved in $\mathrm{CH}_{2} \mathrm{Cl}_{2}(20 \mathrm{~mL})$ and was washed with $\mathrm{H}_{2} \mathrm{O}(10 \mathrm{~mL})$, brine $(10 \mathrm{~mL})$ and dried over $\mathrm{Na}_{2} \mathrm{SO}_{4}$. The organic layer was concentrated in vacuo, and the crude product was purified via flash silica gel chromatography (7:3, $\left.\mathrm{Et}_{2} \mathrm{O}: \mathrm{Hex}\right)$ to afford 2-(2-Bromo-5-methoxy-benzyl)-3-oxopiperidine-1-carboxylic acid methyl ester $(0.70 \mathrm{~g}, 1.95 \mathrm{mmol})$ in $99 \%$ yield. ${ }^{1} \mathrm{H}$ NMR $\left(\mathrm{d}_{6}-\mathrm{DMSO}, 400 \mathrm{MHz}, 363 \mathrm{~K}\right) \delta 1.91-1.99(\mathrm{~m}, 2 \mathrm{H}), 2.40-2.47(\mathrm{~m}, 1 \mathrm{H}), 2.57-2.65(\mathrm{~m}, 1 \mathrm{H})$, 3.12 (dd, 2H, J=7.5 Hz, J=13.5 Hz), 3.33-3.40 (m, 1H), 3.38 (bs, 3H), 3.75 (s, 3H), 3.91$3.97(\mathrm{~m}, 1 \mathrm{H}), 4.74(\mathrm{dd}, 1 \mathrm{H}, \mathrm{J}=6.0 \mathrm{~Hz}, \mathrm{~J}=9.0 \mathrm{~Hz}), 6.76(\mathrm{dd}, 1 \mathrm{H}, \mathrm{J}=3.0 \mathrm{~Hz}, \mathrm{~J}=9.0 \mathrm{~Hz}), 6.84$ $(\mathrm{d}, 1 \mathrm{H}, \mathrm{J}=3.0 \mathrm{~Hz}), 7.44(\mathrm{~d}, 1 \mathrm{H}, \mathrm{J}=9.0 \mathrm{~Hz}) ;{ }^{13} \mathrm{C}$ NMR $\left(\mathrm{d}_{6}-\mathrm{DMSO}, 400 \mathrm{MHz}, 363 \mathrm{~K}\right) \delta 21.7$, 35.9, 35.9, 38.3, 51.4, 54.9, 62.2, 114.1, 114.1, 116.9, 132.3, 136.9, 154.1, 158.1, 205.7; IR ( NaCl) v 1446, 1601, 1700, 2953; MS (ESI) $356\left(\mathrm{M}+\mathrm{H}^{+}\right), 358\left(\mathrm{M}+\mathrm{H}^{+}\right)$.

2-(2-Bromo-5-methoxy-benzyl)-3-oxo-3,6-dihydro-2H-pyridine-1-carboxylic acid methyl ester (13)

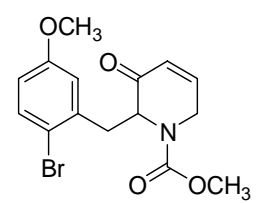

To a solution of 2-(2-Bromo-5-methoxy-benzyl)-3-oxo-piperidine-1-carboxylic acid methyl ester $(63.5 \mathrm{mg}, 0.18 \mathrm{mmol})$ in anhydrous DMSO $(1 \mathrm{~mL})$ was added IBX $(50.0 \mathrm{mg}$, $0.18 \mathrm{mmol}$ ), and the mixture was heated to $70{ }^{\circ} \mathrm{C}$. The reaction was allowed to stir at $70{ }^{\circ} \mathrm{C}$ for $4 \mathrm{~h}$, then an additional equivalent of IBX was added, and after $4 \mathrm{~h}$, a third equivalent of IBX was added and the reaction mixture was allowed to stir at $70{ }^{\circ} \mathrm{C}$ overnight. The mixture was filtered and the filtrate was washed with ether $(3 \mathrm{X}, 3 \mathrm{~mL})$. The organic extracts were combined and washed with sat. $\mathrm{NaHCO}_{3}(3 \mathrm{~mL})$, brine $(3 \mathrm{~mL})$, dried with $\mathrm{MgSO}_{4}$, filtered and concentrated to afford 2-(2-Bromo-5-methoxy-benzyl)-3oxo-3,6-dihydro-2H-pyridine-1-carboxylic acid methyl ester $(60.0 \mathrm{mg}, 0.17 \mathrm{mmol})$ in a $95 \%$ yield. ${ }^{1} \mathrm{H}$ NMR (d 6 -DMSO, $\left.400 \mathrm{MHz}, 363 \mathrm{~K}\right) \delta 2.94-3.04(\mathrm{~m}, 2 \mathrm{H}), 3.39(\mathrm{bs}, 3 \mathrm{H})$, 3.75 (s, 3H), 4.10 (ddd, 1H, J=2.5 Hz, J=2.5 Hz, J=11.0 Hz), 4.55 (ddd, 1H, J=2.0 Hz, $\mathrm{J}=5.0 \mathrm{~Hz}, \mathrm{~J}=21.0 \mathrm{~Hz}), 4.85(\mathrm{dd}, 1 \mathrm{H}, \mathrm{J}=5.5 \mathrm{~Hz}, \mathrm{~J}=9.5 \mathrm{~Hz}), 6.10(\mathrm{dt}, 1 \mathrm{H}, \mathrm{J}=2.5 \mathrm{~Hz}, \mathrm{~J}=10.5$ $\mathrm{Hz}$ ), 6.77 (dd, 1H, J=3.0 Hz, J=9.0 Hz), 6.84 (d, 1H, J=3.0 Hz), 7.20 (ddd, 1H, J=2.0 Hz, $\mathrm{J}=5.0 \mathrm{~Hz}, \mathrm{~J}=10.0 \mathrm{~Hz}), 7.45(\mathrm{~d}, 1 \mathrm{H}, 9.0 \mathrm{~Hz}) ;{ }^{13} \mathrm{C} \mathrm{NMR}\left(\mathrm{d}_{6}-\mathrm{DMSO}, 400 \mathrm{MHz}, 363 \mathrm{~K}\right) \delta$ 35.8, 51.6, 54.9, 59.3, 62.2, 114.1, 114.2, 116.9, 124.6, 132.4, 136.4, 146.9, 154.3, 158.1, 193.0; IR ( NaCl) v 1446, 1595, 1688, 1705, 2953; MS (ESI) $354\left(\mathrm{M}+\mathrm{H}^{+}\right), 356\left(\mathrm{M}+\mathrm{H}^{+}\right)$. 


\section{2-(2-Bromo-5-methoxy-benzyl)-3-hydroxy-3-(3-methoxy-phenyl)-3,6-dihydro-2H- pyridine-1-carboxylic acid methyl ester (14)}

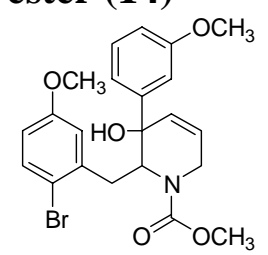

To a solution of 2-(2-Bromo-5-methoxy-benzyl)-3-oxo-3,6-dihydro-2H-pyridine-1carboxylic acid methyl ester $(40.0 \mathrm{mg}, 0.11 \mathrm{mmol})$ in anhydrous THF $(3 \mathrm{~mL})$ was added 3-methoxyphenylmagnesium bromide $(226 \mu \mathrm{L}, 0.22 \mathrm{mmol})$ dropwise at $\mathrm{rt}$. The reaction was quenched with $\mathrm{H}_{2} \mathrm{O}(2 \mathrm{~mL})$ after an hour and extracted with $\mathrm{Et}_{2} \mathrm{O}(3 \mathrm{X}, 5 \mathrm{~mL})$. The organic layers were combined, dried with $\mathrm{MgSO}_{4}$, filtered and concentrated in vacuo. The crude product was purified via flash silica gel chromatography $\left(3: 2, \mathrm{Et}_{2} \mathrm{O}: \mathrm{Hex}\right)$ to afford 2-(2-Bromo-5-methoxy-benzyl)-3-hydroxy-3-(3-methoxy-phenyl)-3,6-dihydro$2 \mathrm{H}$-pyridine-1-carboxylic acid methyl ester $(39.0 \mathrm{mg}, 0.08 \mathrm{mmol})$ in a $76 \%$ yield. ${ }^{1} \mathrm{H}$

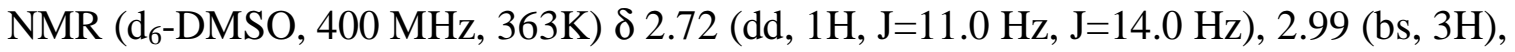
3.47 (dd, 1H, J=3.0 Hz, J=14.0 Hz), 3.72-3.75 (m, 1H), $3.73(\mathrm{~s}, 3 \mathrm{H}), 3.74(\mathrm{~m}, 3 \mathrm{H}), 4.16$ (ddd, 1H, J=3.0 Hz, J=3.0 Hz, J=19.0 Hz), $4.63(\mathrm{~d}, 1 \mathrm{H}, \mathrm{J}=10.5 \mathrm{~Hz}$ ), 5.47 (bs, 1H), 5.80$5.86(\mathrm{~m}, 1 \mathrm{H}), 6.01-6.03(\mathrm{~m}, 1 \mathrm{H}), 6.70(\mathrm{dd}, 1 \mathrm{H}, \mathrm{J}=3.0 \mathrm{~Hz}, \mathrm{~J}=9.0 \mathrm{~Hz}), 6.78$ (ddd, J=1.0 Hz, $\mathrm{J}=2.5 \mathrm{~Hz}, \mathrm{~J}=8.0 \mathrm{~Hz}), 6.86(\mathrm{~d}, 1 \mathrm{H}, \mathrm{J}=3.0 \mathrm{~Hz}), 6.99-7.02(\mathrm{~m}, 2 \mathrm{H}), 7.19(\mathrm{t}, 1 \mathrm{H}, \mathrm{J}=8.0 \mathrm{~Hz})$, $7.40(\mathrm{~d}, 1 \mathrm{H}, \mathrm{J}=9.0 \mathrm{~Hz}) ;{ }^{13} \mathrm{C}$ NMR $\left(\mathrm{d}_{6}\right.$-DMSO, $\left.400 \mathrm{MHz}, 363 \mathrm{~K}\right) \delta 33.3,50.9,54.5,54.9$, 58.5, 58.7, 72.3, 111.6, 111.8, 113.6, 114.3, 116.4, 117.8, 122.7, 127.8, 129.6, 132.2, 139.2, 147.5, 154.6, 158.1, 158.5; IR ( NaCl) v 1456, 1597, 1653, 1684, 2952, 3420; MS (ESI) $444\left(\mathrm{M}+\mathrm{H}^{+}\right), 446\left(\mathrm{M}+\mathrm{H}^{+}\right)$.

\section{6-Bromo-3-methoxy-13-(3-methoxy-phenyl)-10-aza-tricyclo[7.2.2.02,7]trideca-} 2(7),3,5,12-tetraene-10-carboxylic acid methyl ester (15)

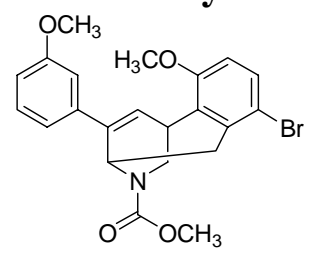

A solution of 2-(2-Bromo-5-methoxy-benzyl)-3-hydroxy-3-(3-methoxy-phenyl)-3,6dihydro-2H-pyridine-1-carboxylic acid methyl ester $(27.0 \mathrm{mg}, 0.06 \mathrm{mmol})$ in anhydrous $\mathrm{CH}_{2} \mathrm{Cl}_{2}(1 \mathrm{~mL})$ was added dropwise to a $\mathrm{CF}_{3} \mathrm{SO}_{3} \mathrm{H}(1 \mathrm{~mL})$ and $\mathrm{CH}_{2} \mathrm{Cl}_{2}(1 \mathrm{~mL})$ mixture at $-30{ }^{\circ} \mathrm{C}$. The reaction mixture was allowed to stir at $-30{ }^{\circ} \mathrm{C}$ for $30 \mathrm{~min}$ then poured into $\mathrm{H}_{2} \mathrm{O}(10 \mathrm{~mL})$ and extracted with $\mathrm{CH}_{2} \mathrm{Cl}_{2}(3 \mathrm{X}, 25 \mathrm{~mL})$. The organic layers were combined, washed with sat. $\mathrm{NaHCO}_{3}(30 \mathrm{~mL})$, dried with $\mathrm{Na}_{2} \mathrm{SO}_{4}$ and concentrated in vacuo to afford 6-Bromo-3-methoxy-13-(3-methoxy-phenyl)-10-azatricyclo[7.2.2.02,7]trideca-2(7),3,5,12-tetraene-10-carboxylic acid methyl ester (22.0 mg, $0.05 \mathrm{mmol}$ ) in a $81 \%$ yield with only one isomer observed by NMR. ${ }^{1} \mathrm{H}$ NMR $\left(\mathrm{d}_{6}\right.$-DMSO, $400 \mathrm{MHz}, 363 \mathrm{~K}) \delta 2.85(\mathrm{dd}, 1 \mathrm{H}, \mathrm{J}=2.5 \mathrm{~Hz}, \mathrm{~J}=18.0 \mathrm{~Hz}), 3.21(\mathrm{dd}, 1 \mathrm{H}, \mathrm{J}=4.5 \mathrm{~Hz}, \mathrm{~J}=18.0$ $\mathrm{Hz}$ ), 3.48 (dd, $1 \mathrm{H}, \mathrm{J}=4.5 \mathrm{~Hz}, \mathrm{~J}=11.0 \mathrm{~Hz}), 3.65-3.67(\mathrm{~m}, 1 \mathrm{H}), 3.65$ (bs, 3H), $3.80(\mathrm{~s}, 3 \mathrm{H})$, $3.84(\mathrm{~s}, 3 \mathrm{H}), 4.53$ (ddd, 1H, J=1.5 Hz, J=4.0 Hz, J=8.0 Hz), 5.43 (bs, 1H), 6.82 (dd, 1H, 
$\mathrm{J}=1.0 \mathrm{~Hz}, \mathrm{~J}=8.0 \mathrm{~Hz}), 6.87(\mathrm{~d}, 1 \mathrm{H}, 9.0 \mathrm{~Hz}), 6.88-6.91(\mathrm{~m}, 1 \mathrm{H}), 7.01(\mathrm{~m}, 1 \mathrm{H})$, 7.04-7.07 (m, $1 \mathrm{H}), 7.31(\mathrm{t}, 1 \mathrm{H}, \mathrm{J}=8.0 \mathrm{~Hz}), 7.43(\mathrm{~d}, 1 \mathrm{H}, \mathrm{J}=9.0 \mathrm{~Hz}) ;{ }^{13} \mathrm{C} \mathrm{NMR}\left(\mathrm{d}_{6}-\mathrm{DMSO}, 400 \mathrm{MHz}\right.$, $363 \mathrm{~K}) \delta 28.7,38.4,48.8,50.2,51.7,54.7,56.0,110.1,111.1,111.2,113.1,116.7,116.73$, $128.3,129.2$, 130.4, 130.7, 134.9, 137.6, 139.6, 154.6, 159.3; IR $(\mathrm{NaCl})$ v 1452, 1577, 1697, 2926; HRMS (EM) $[\mathrm{M}+\mathrm{H}]^{+}$calcd for $\mathrm{C}_{22} \mathrm{H}_{22} \mathrm{BrNO}_{4} \mathrm{Na} 466.0624$, found 466.0609.

\section{2-(2-Bromo-5-methoxy-benzyl)-3-hydroxy-3-(3-methoxy-phenyl)-piperidine-1-} carboxylic acid methyl ester (16)

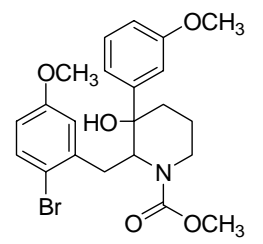

To a solution of 2-(2-Bromo-5-methoxy-benzyl)-3-oxo-piperidine-1-carboxylic acid methyl ester $(18.6 \mathrm{mg}, 0.05 \mathrm{mmol})$ in anhydrous THF $(300 \mu \mathrm{L})$ was added 3methoxyphenylmagnesium bromide $(61.0 \mu \mathrm{L}, 0.06 \mathrm{mmol})$ dropwise at $\mathrm{rt}$. The reaction was quenched with $\mathrm{H}_{2} \mathrm{O}(2 \mathrm{~mL})$ after an hour, and extracted with $\mathrm{Et}_{2} \mathrm{O}(3 \mathrm{X}, 5 \mathrm{~mL})$. The organic layers were combined, dried with $\mathrm{MgSO}_{4}$, filtered and concentrated in vacuo. The crude product was purified via flash silica gel chromatography $\left(3: 2, \mathrm{Et}_{2} \mathrm{O}: \mathrm{Hex}\right)$ to afford 2-(2-Bromo-5-methoxy-benzyl)-3-hydroxy-3-(3-methoxy-phenyl)-piperidine-1carboxylic acid methyl ester $(15.0 \mathrm{mg}, 0.03 \mathrm{mmol})$ in a $65 \%$ yield. ${ }^{1} \mathrm{H}$ NMR $\left(\mathrm{d}_{6}\right.$-DMSO, $400 \mathrm{MHz}, 363 \mathrm{~K}) \delta 1.25-1.34(\mathrm{~m}, 1 \mathrm{H}), 1.60-1.64(\mathrm{~m}, 1 \mathrm{H}), 1.95-2.12(\mathrm{~m}, 2 \mathrm{H}), 3.09(\mathrm{dd}$, $1 \mathrm{H}, \mathrm{J}=11.0 \mathrm{~Hz}, \mathrm{~J}=14.0 \mathrm{~Hz}), 3.18(\mathrm{~s}, 3 \mathrm{H}), 3.24(\mathrm{dd}, 1 \mathrm{H}, \mathrm{J}=4.0 \mathrm{~Hz}, \mathrm{~J}=13.0 \mathrm{~Hz}), 3.83$ (dd, $1 \mathrm{H}, \mathrm{J}=6.0 \mathrm{~Hz}, \mathrm{~J}=13.0 \mathrm{~Hz}), 4.95(\mathrm{dd}, \mathrm{J}=3.0 \mathrm{~Hz}, \mathrm{~J}=11.0 \mathrm{~Hz}), 5.07$ (bs, 1H), 6.70 (dd, 1H, $\mathrm{J}=3.0 \mathrm{~Hz}, \mathrm{~J}=9.0 \mathrm{~Hz}), 6.80(\mathrm{ddd}, 1 \mathrm{H}, \mathrm{J}=1.0 \mathrm{~Hz}, \mathrm{~J}=2.5 \mathrm{~Hz}, \mathrm{~J}=8.0 \mathrm{~Hz}), 6.88(\mathrm{~d}, 1 \mathrm{H}, \mathrm{J}=3.0$ $\mathrm{Hz}), 7.12-7.14(\mathrm{~m}, 2 \mathrm{H}), 7.22(\mathrm{t}, 1 \mathrm{H}, \mathrm{J}=8.0 \mathrm{~Hz}), 7.39(\mathrm{~d}, 1 \mathrm{H}, \mathrm{J}=9.0 \mathrm{~Hz}) ;{ }^{13} \mathrm{C} \mathrm{NMR}\left(\mathrm{d}_{6^{-}}\right.$ DMSO, $400 \mathrm{MHz}, 363 \mathrm{~K}) \delta 21.1,31.6,32.3$, 37.4, 51.0, 54.5, 54.9, 58.2, 71.8, 111.8, $113.5,114.5,116.9,118.0,128.1,132.0,139.5,147.7,154.6,158.0,158.6 ; \mathrm{IR}(\mathrm{NaCl}) v$

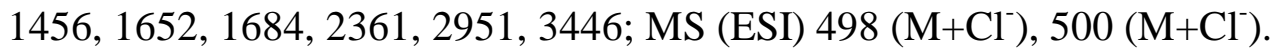




\section{8-Bromo-5-methoxy-4 $\alpha$-(3-methoxy-phenyl)-2,3,4,4 $\alpha, 9,9 \alpha$-hexahydro-indeno[2,1- B]pyridine-1-carboxylic acid methyl ester (17)}

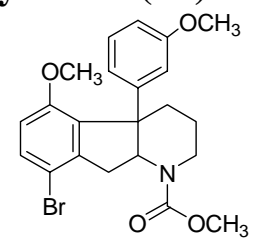

A solution of 2-(2-Bromo-5-methoxy-benzyl)-3-hydroxy-3-(3-methoxy-phenyl)piperidine-1-carboxylic acid methyl ester $(22.0 \mathrm{mg}, 0.05 \mathrm{mmol})$ in anhydrous $\mathrm{CH}_{2} \mathrm{Cl}_{2}$ (1 $\mathrm{mL}$ ) was added dropwise to a $\mathrm{CF}_{3} \mathrm{SO}_{3} \mathrm{H}(1 \mathrm{~mL})$ and $\mathrm{CH}_{2} \mathrm{Cl}_{2}(1 \mathrm{~mL})$ mixture at $-30{ }^{\circ} \mathrm{C}$. The reaction mixture was allowed to stir at $-30{ }^{\circ} \mathrm{C}$ for $30 \mathrm{~min}$, then allowed to warm to $\mathrm{rt}$ and stir 2 hours. The reaction mixture was then poured into $\mathrm{H}_{2} \mathrm{O}(10 \mathrm{~mL})$ and extracted with $\mathrm{CH}_{2} \mathrm{Cl}_{2}(3 \mathrm{X}, 25 \mathrm{~mL})$. The organic layers were combined, washed with sat. $\mathrm{NaHCO}_{3}$ $\left(30 \mathrm{~mL}\right.$ ), dried with $\mathrm{Na}_{2} \mathrm{SO}_{4}$ and concentrated in vacuo to afford 8-Bromo-5-methoxy$4 \alpha$-(3-methoxy-phenyl)-2,3,4,4 $\alpha, 9,9 \alpha$-hexahydro-indeno[2,1- $\beta$ ]pyridine-1-carboxylic acid methyl ester $(16.0 \mathrm{mg}, 0.04 \mathrm{mmol})$ in a $77 \%$ yield. ${ }^{1} \mathrm{H}$ NMR $\left(\mathrm{d}_{6}-\mathrm{DMSO}, 400 \mathrm{MHz}\right.$, $363 \mathrm{~K}) \delta 1.38-1.47(\mathrm{~m}, 1 \mathrm{H}), 1.73-1.83(\mathrm{~m}, 1 \mathrm{H}), 2.01-2.08(\mathrm{~m}, 1 \mathrm{H}), 2.38-2.44(\mathrm{~m}, 1 \mathrm{H})$, 2.94-3.00 (m, 1H), $3.21(\mathrm{dd}, 1 \mathrm{H}, \mathrm{J}=8.5 \mathrm{~Hz}, \mathrm{~J}=17.0 \mathrm{~Hz}), 3.46(\mathrm{~s}, 3 \mathrm{H}), 3.57$ (s, 3H), 3.72 (s, $3 \mathrm{H}), 3.83-3.90(\mathrm{~m}, 1 \mathrm{H}), 4.99(\mathrm{t}, 1 \mathrm{H}, \mathrm{J}=8.0 \mathrm{~Hz}), 6.74-6.81(\mathrm{~m}, 4 \mathrm{H}), 7.19(\mathrm{t}, 1 \mathrm{H}, \mathrm{J}=8.0 \mathrm{~Hz})$, $7.40(\mathrm{~d}, 1 \mathrm{H}, \mathrm{J}=9.0 \mathrm{~Hz}) ;{ }^{13} \mathrm{C}$ NMR $\left(\mathrm{d}_{6}\right.$-DMSO, $\left.400 \mathrm{MHz}, 363 \mathrm{~K}\right) \delta 19.1,27.1,35.7,37.6$, 51.6, 53.9, 54.5, 55.1, 61.7, 109.4, 110.6, 112.6, 112.7, 118.5, 128.2, 130.7, 136.9, 141.0, 147.0, 154.9, 155.2, 158.7; IR (NaCl) v 1446, 1471, 1600, 1695, 2927; MS (ESI) 446 $\left(\mathrm{M}+\mathrm{H}^{+}\right), 448\left(\mathrm{M}+\mathrm{H}^{+}\right)$.

\section{2-(2-Bromo-5-methoxy-benzyl)-5-(3-methoxy-phenyl)-3-oxo-3,6-dihydro-2H- pyridine-1-carboxylic acid methyl ester (18)}

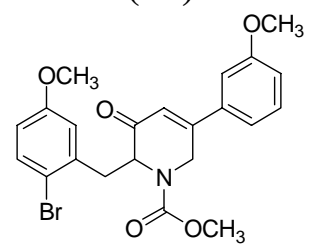

To a solution of $\mathrm{CuBrSMe}(6.0 \mathrm{mg}, 0.03 \mathrm{mmol})$ in anhydrous $\mathrm{THF}(350 \mu \mathrm{L})$ at $-78{ }^{\circ} \mathrm{C}$ was added 3-methoxyphenylmagnesium bromide $(207 \mu \mathrm{L}, 0.21 \mathrm{mmol})$ dropwise, and allowed to stir at $-78{ }^{\circ} \mathrm{C}$ for $30 \mathrm{~min}$. Next, 2-(2-Bromo-5-methoxy-benzyl)-3-oxo-3,6dihydro-2H-pyridine-1-carboxylic acid methyl ester $(49.0 \mathrm{mg}, 0.14 \mathrm{mmol})$ in anhydrous THF $(500 \mu \mathrm{L})$ was added dropwise via syringe, and the reaction was allowed to stir at $-78{ }^{\circ} \mathrm{C}$ for $30 \mathrm{~min}$. Next, phenylselenyl chloride $(45.0 \mathrm{mg}, 0.24 \mathrm{mmol})$ and diphenyl diselenide (few crystals) in anhydrous THF $(500 \mu \mathrm{L})$ was added dropwise, and the reaction mixture was allowed to stir at $-78^{\circ} \mathrm{C}$ for $30 \mathrm{~min}$. The reaction mixture was then allowed to warm to $0{ }^{\circ} \mathrm{C}$, and a mixture of acetic acid $(31.0 \mu \mathrm{L})$ in $\mathrm{H}_{2} \mathrm{O}(156 \mu \mathrm{L})$ was added dropwise followed by a $30 \% \mathrm{H}_{2} \mathrm{O}_{2}(156 \mu \mathrm{L})$. The mixture was then allowed to warm to rt, and after $10 \mathrm{~min}$ the complete reaction mixture was added to sat. $\mathrm{NaHCO}_{3}(5$ $\mathrm{mL})$ and extracted with $\mathrm{Et}_{2} \mathrm{O}(3 \mathrm{X}, 10 \mathrm{~mL})$. The organic layers were combined and 
washed with sat. $\mathrm{NH}_{4} \mathrm{Cl}(15 \mathrm{~mL})$ and brine $(15 \mathrm{~mL})$, dried with $\mathrm{MgSO}_{4}$, filtered, and concentrated in vacuo. The crude product was purified via flash silica gel chromatography $\left(1: 1, \quad \mathrm{Et}_{2} \mathrm{O}: \mathrm{Hex}\right)$ to afford 2-(2-Bromo-5-methoxy-benzyl)-5-(3methoxy-phenyl)-3-oxo-3,6-dihydro-2H-pyridine-1-carboxylic acid methyl ester (36.0 $\mathrm{mg}, 0.08 \mathrm{mmol})$ in a $57 \%$ yield. ${ }^{1} \mathrm{H}$ NMR $\left(\mathrm{d}_{6}-\mathrm{DMSO}, 400 \mathrm{MHz}, 363 \mathrm{~K}\right) \delta 3.10(\mathrm{dd}, 1 \mathrm{H}$, $\mathrm{J}=8.0 \mathrm{~Hz}, \mathrm{~J}=8.0 \mathrm{~Hz}$ ), 3.42 (bs, 3H), $3.76(\mathrm{~s}, 3 \mathrm{H}), 3.86(\mathrm{~s}, 3 \mathrm{H}), 4.52(\mathrm{dd}, 1 \mathrm{H}, \mathrm{J}=2.5 \mathrm{~Hz}$, $\mathrm{J}=20.0 \mathrm{~Hz}), 4.92(\mathrm{dd}, 1 \mathrm{H}, \mathrm{J}=5.5 \mathrm{~Hz}, \mathrm{~J}=9.5 \mathrm{~Hz}), 4.98(\mathrm{dd}, 1 \mathrm{H}, \mathrm{J}=1.0 \mathrm{~Hz}, \mathrm{~J}=20.0, \mathrm{~Hz})$, 6.52-6.53 (m, 1H), 6.78 (dd, 1H, J=3.0 Hz, J=9.0 Hz), 6.94 (d, 1H, J=3.0 Hz), 7.10 (dd, $1 \mathrm{H}, \mathrm{J}=2.5 \mathrm{~Hz}, \mathrm{~J}=8.0 \mathrm{~Hz}), 7.21-7.22(\mathrm{~m}, 1 \mathrm{H}), 7.27(\mathrm{ddd}, 1 \mathrm{H}, \mathrm{J}=1.0 \mathrm{~Hz}, \mathrm{~J}=1.5 \mathrm{~Hz}, \mathrm{~J}=8.0$ $\mathrm{Hz}), 7.42(\mathrm{t}, 1 \mathrm{H}, \mathrm{J}=8.0 \mathrm{~Hz}), 7.46(\mathrm{~d}, 1 \mathrm{H}, \mathrm{J}=9.0 \mathrm{~Hz}) ;{ }^{13} \mathrm{C}$ NMR $\left(\mathrm{d}_{6}-\mathrm{DMSO}, 400 \mathrm{MHz}\right.$, $363 \mathrm{~K}) \delta 35.4,41.3,51.8,54.9,55.0,58.5,111.4,114.2,114.2,116.2,117.1,118.1,120.9$, 129.5, 132.4, 136.1, 136.6, 154.5, 155.2, 158.2, 159.3, 193.2; IR ( NaCl) v 1448, 1576, 1670, 1704, 2953; MS (ESI) $460\left(\mathrm{M}+\mathrm{H}^{+}\right), 462\left(\mathrm{M}+\mathrm{H}^{+}\right)$.

\section{2-(2-Bromo-5-methoxy-benzyl)-3-hydroxy-3,5-bis-(3-methoxy-phenyl)-3,6-dihydro- 2H-pyridine-1-carboxylic acid methyl ester (19)}

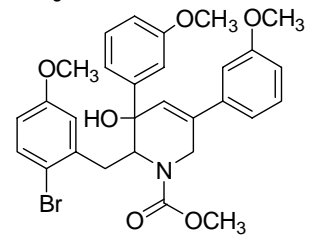

To a solution of 2-(2-Bromo-5-methoxy-benzyl)-5-(3-methoxy-phenyl)-3-oxo-3,6dihydro-2H-pyridine-1-carboxylic acid methyl ester $(30.0 \mathrm{mg}, 0.06 \mathrm{mmol})$ in anhydrous THF $(500 \mu \mathrm{L})$ was added 3-methoxyphenylmagnesium bromide $(130.0 \mu \mathrm{L}, 0.13 \mathrm{mmol})$ dropwise at $\mathrm{rt}$. The reaction was quenched with $\mathrm{H}_{2} \mathrm{O}(2 \mathrm{~mL})$ after an hour, and extracted with $\mathrm{Et}_{2} \mathrm{O}(3 \mathrm{X}, 5 \mathrm{~mL})$. The organic layers were combined, dried with $\mathrm{MgSO}_{4}$, filtered and concentrated in vacuo. The crude product was purified via flash silica gel chromatography (1:1, $\left.\mathrm{Et}_{2} \mathrm{O}: \mathrm{Hex}\right)$ to afford 2-(2-Bromo-5-methoxy-benzyl)-3-hydroxy3,5-bis-(3-methoxy-phenyl)-3,6-dihydro-2H-pyridine-1-carboxylic acid methyl ester (23.0 mg, $0.04 \mathrm{mmol}$ ) in a 62\% yield. ${ }^{1} \mathrm{H}$ NMR $\left(\mathrm{d}_{6}-\mathrm{DMSO}, 400 \mathrm{MHz}, 363 \mathrm{~K}\right) \delta 2.81(\mathrm{dd}$, $1 \mathrm{H}, \mathrm{J}=11.0 \mathrm{~Hz}, \mathrm{~J}=14.5 \mathrm{~Hz}$ ), 3.08 (bs, 3H), 3.51 (dd, 1H, J=3.5 Hz, J=14.5 Hz), 3.74 (s, $3 \mathrm{H}), 3.84(\mathrm{~s}, 3 \mathrm{H}), 4.07$ (dd, 1H, J=1.5 Hz, J=18.5 Hz), $4.55(\mathrm{dd}, 1 \mathrm{H}, \mathrm{J}=0.5 \mathrm{~Hz}, \mathrm{~J}=19.0$ $\mathrm{Hz}), 4.72(\mathrm{~d}, 1 \mathrm{H}, \mathrm{J}=9.5 \mathrm{~Hz}), 6.20(\mathrm{~m}, 1 \mathrm{H}), 6.72(\mathrm{dd}, 1 \mathrm{H}, \mathrm{J}=3.0 \mathrm{~Hz}, \mathrm{~J}=9.0 \mathrm{~Hz}), 6.80(\mathrm{~m}$, $1 \mathrm{H}), 6.93(\mathrm{~d}, 1 \mathrm{H}, \mathrm{J}=3.0 \mathrm{~Hz}), 6.96(\mathrm{dd}, 1 \mathrm{H}, \mathrm{J}=2.5 \mathrm{~Hz}, \mathrm{~J}=8.0 \mathrm{~Hz}), 7.01-7.04(\mathrm{~m}, 2 \mathrm{H}), 7.09-$ $7.10(\mathrm{~m}, 1 \mathrm{H}), 7.15(\mathrm{~d}, 1 \mathrm{H}, \mathrm{J}=8.0 \mathrm{~Hz}), 7.20(\mathrm{t}, 1 \mathrm{H}, \mathrm{J}=8.0 \mathrm{~Hz}), 7.36(\mathrm{t}, 1 \mathrm{H}, \mathrm{J}=8.0 \mathrm{~Hz}), 7.42$ $(\mathrm{d}, 1 \mathrm{H}, \mathrm{J}=9.0 \mathrm{~Hz}) ;{ }^{13} \mathrm{C}$ NMR $\left(\mathrm{d}_{6}\right.$-DMSO, $\left.400 \mathrm{MHz}, 363 \mathrm{~K}\right) \delta 33.2,41.2,51.1,54.5,54.8$, 54.9, 58.5, 72.6, 110.8, 111.6, 111.9, 113.2, 113.7, 114.3, 116.5, 117.2, 117.9, 127.1, $127.9,129.1,132.2,132.5,138.5,139.0,147.6,158.1,158.5,159.2 ; \mathrm{IR}(\mathrm{NaCl})$ v 1450, 1481, 1598, 1697, 2953, 3394; HRMS (EM) $[\mathrm{M}+\mathrm{H}]^{+}$calcd for $\mathrm{C}_{29} \mathrm{H}_{30} \mathrm{BrNO}_{6} \mathrm{Na} 590.1148$, found 590.1145 . 


\section{8-Bromo-5-methoxy-3,4 $\alpha$-bis-(3-methoxy-phenyl)-2,4 $\alpha, 9,9 \alpha$-tetrahydro-indeno[2,1-} $\beta$ ]pyridine-1-carboxylic acid methyl ester (20)

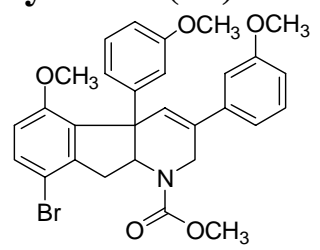

A solution of 2-(2-Bromo-5-methoxy-benzyl)-3-hydroxy-3,5-bis-(3-methoxy-phenyl)3,6-dihydro-2H-pyridine-1-carboxylic acid methyl ester $(16.0 \mathrm{mg}, 0.03 \mathrm{mmol})$ in anhydrous $\mathrm{CH}_{2} \mathrm{Cl}_{2}(1 \mathrm{~mL})$ was added dropwise to a $\mathrm{CF}_{3} \mathrm{SO}_{3} \mathrm{H}(1 \mathrm{~mL})$ and $\mathrm{CH}_{2} \mathrm{Cl}_{2}(1 \mathrm{~mL})$ mixture at $-30{ }^{\circ} \mathrm{C}$. The reaction mixture was allowed to stir at $-30{ }^{\circ} \mathrm{C}$ for $30 \mathrm{~min}$ then allowed to warm to rt, and stir 2 hours. The reaction mixture was then poured into $\mathrm{H}_{2} \mathrm{O}$ $(10 \mathrm{~mL})$ and extracted with $\mathrm{CH}_{2} \mathrm{Cl}_{2}(3 \mathrm{X}, 25 \mathrm{~mL})$. The organic layers were combined, washed with sat. $\mathrm{NaHCO}_{3}(30 \mathrm{~mL})$, dried with $\mathrm{Na}_{2} \mathrm{SO}_{4}$, and concentrated in vacuo to afford 8-Bromo-5-methoxy-3,4 $\alpha$-bis-(3-methoxy-phenyl)-2,4 $\alpha, 9,9 \alpha$-tetrahydroindeno[2,1- $\beta$ ]pyridine-1-carboxylic acid methyl ester $(11.0 \mathrm{mg}, 0.02 \mathrm{mmol})$ in a $71 \%$ yield. ${ }^{1} \mathrm{H}$ NMR $\left(\mathrm{d}_{6}\right.$-DMSO, $\left.400 \mathrm{MHz}, 363 \mathrm{~K}\right) \delta 3.00-3.19(\mathrm{~m}, 2 \mathrm{H}), 3.52(\mathrm{~s}, 3 \mathrm{H}), 3.54(\mathrm{~s}$, $3 \mathrm{H}), 3.70(\mathrm{~s}, 3 \mathrm{H}), 3.79(\mathrm{~s}, 3 \mathrm{H}), 4.24(\mathrm{~d}, 1 \mathrm{H}, \mathrm{J}=18.0 \mathrm{~Hz}), 4.66(\mathrm{~d}, 1 \mathrm{H}, \mathrm{J}=18.0 \mathrm{~Hz}), 4.93(\mathrm{t}$, $1 \mathrm{H}, \mathrm{J}=9.0 \mathrm{~Hz}), 6.18(\mathrm{~s}, 1 \mathrm{H}), 6.66(\mathrm{~m}, 1 \mathrm{H}), 6.72(\mathrm{~d}, 1 \mathrm{H}, \mathrm{J}=8.0 \mathrm{~Hz}), 6.80(\mathrm{~d}, 1 \mathrm{H}, \mathrm{J}=8.0 \mathrm{~Hz})$, 6.87-6.96 (m, 3H), $7.03(\mathrm{~d}, 1 \mathrm{H}, \mathrm{J}=8.0 \mathrm{~Hz}), 7.21(\mathrm{t}, 1 \mathrm{H}, \mathrm{J}=8.0 \mathrm{~Hz}), 7.30$ (t, 1H, J=8.0 Hz), $7.44(\mathrm{~d}, 1 \mathrm{H}, \mathrm{J}=9.0 \mathrm{~Hz}) ;{ }^{13} \mathrm{C} \mathrm{NMR}\left(\mathrm{d}_{6}-\mathrm{DMSO}, 400 \mathrm{MHz}, 363 \mathrm{~K}\right) \delta 33.6,40.4,51.8,54.5$, 54.74, 55.13, 55.84, 60.93, 109.61, 110.68, 111.04, 111.85, 112.82, 112.92, 117.06, 117.9, $122.8,128.4,129.1,131.3,132.8,133.1,139.3,140.9,146.3,154.8,154.9,158.7,159.2$; IR $(\mathrm{NaCl})$ v $1447,1703,2850,2920$; HRMS (EM) $[\mathrm{M}+\mathrm{H}]^{+}$calcd for $\mathrm{C}_{29} \mathrm{H}_{28} \mathrm{BrNO}_{5} \mathrm{Na}$ 572.1043 , found 572.1063. 
S11
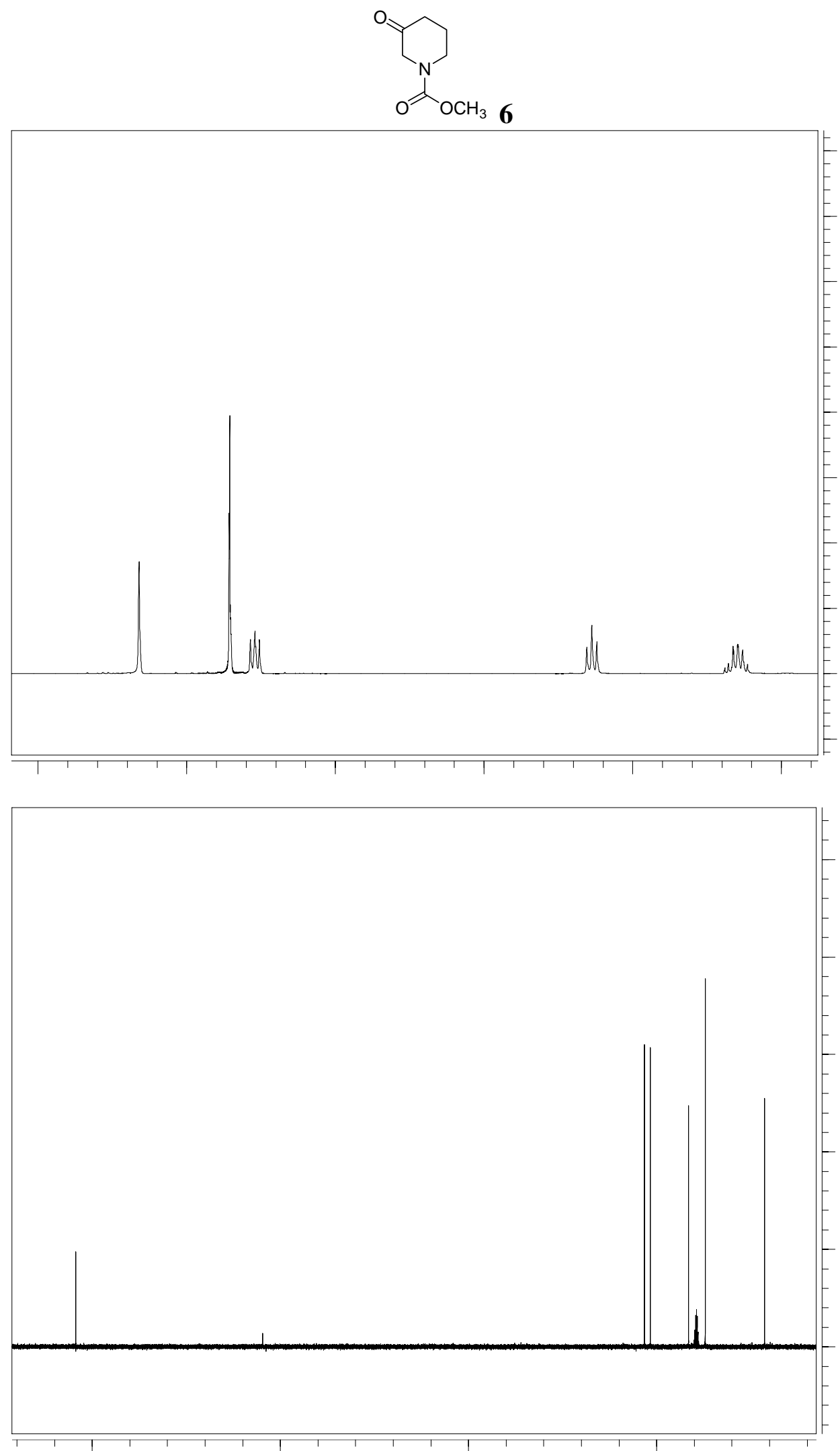

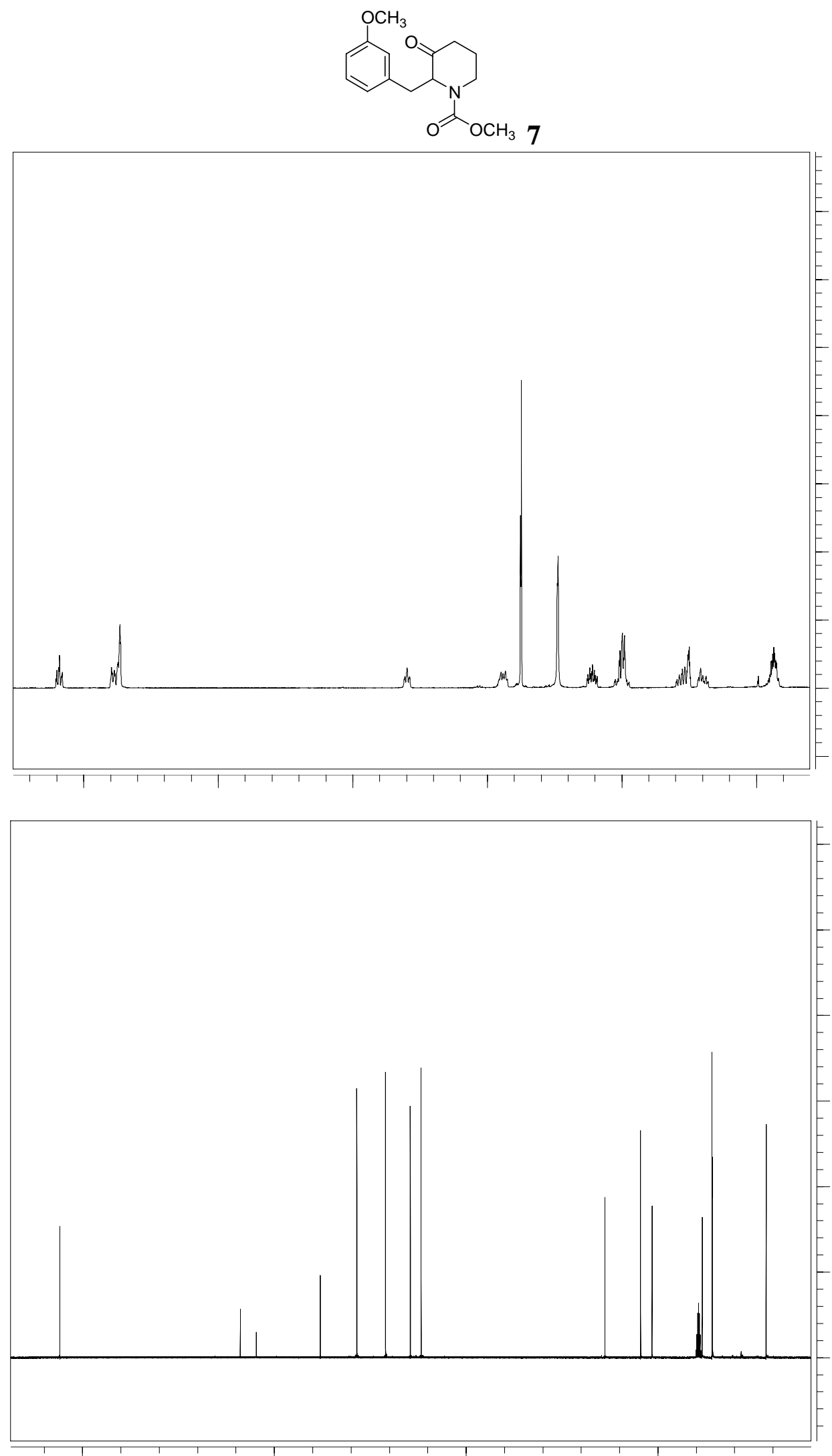

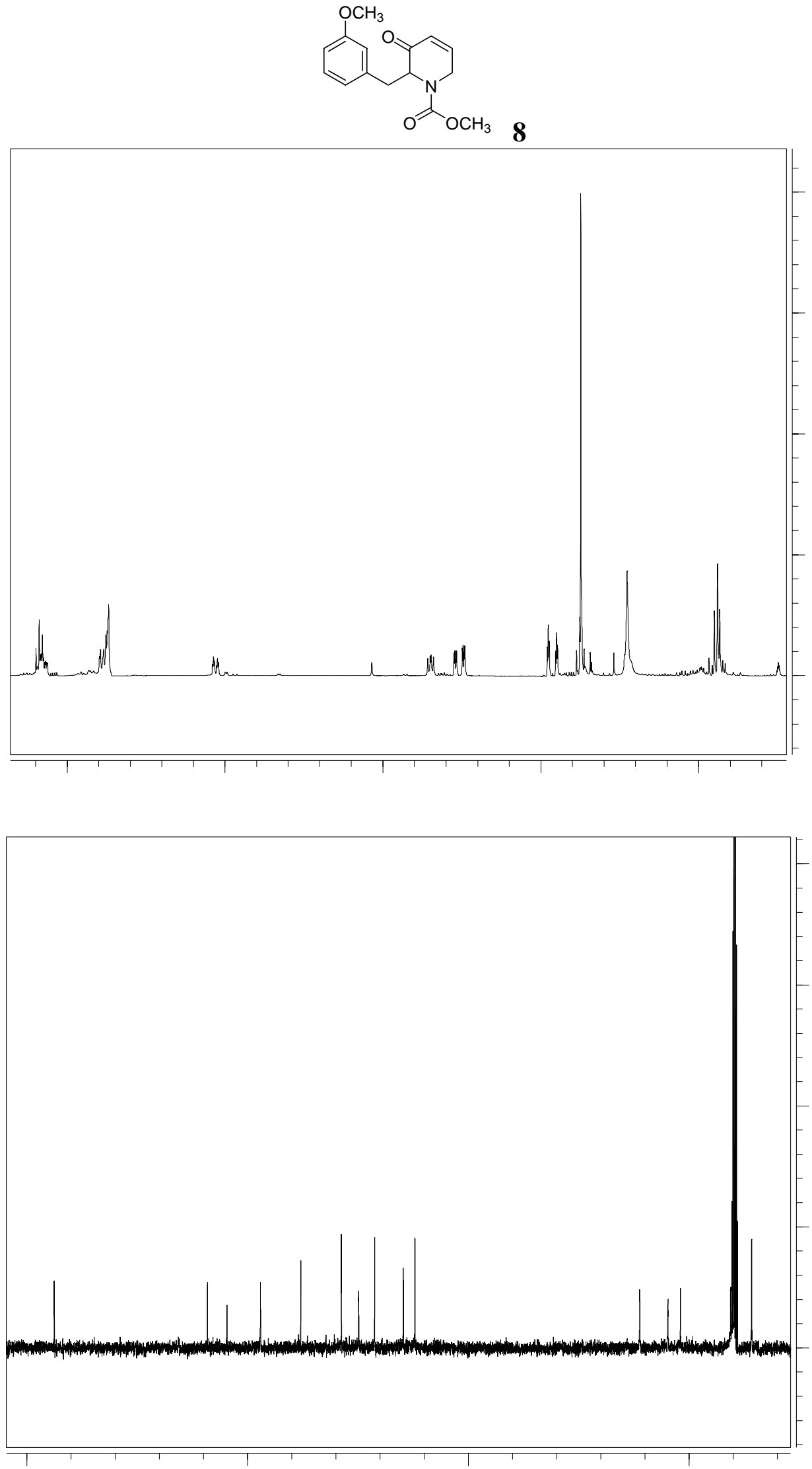

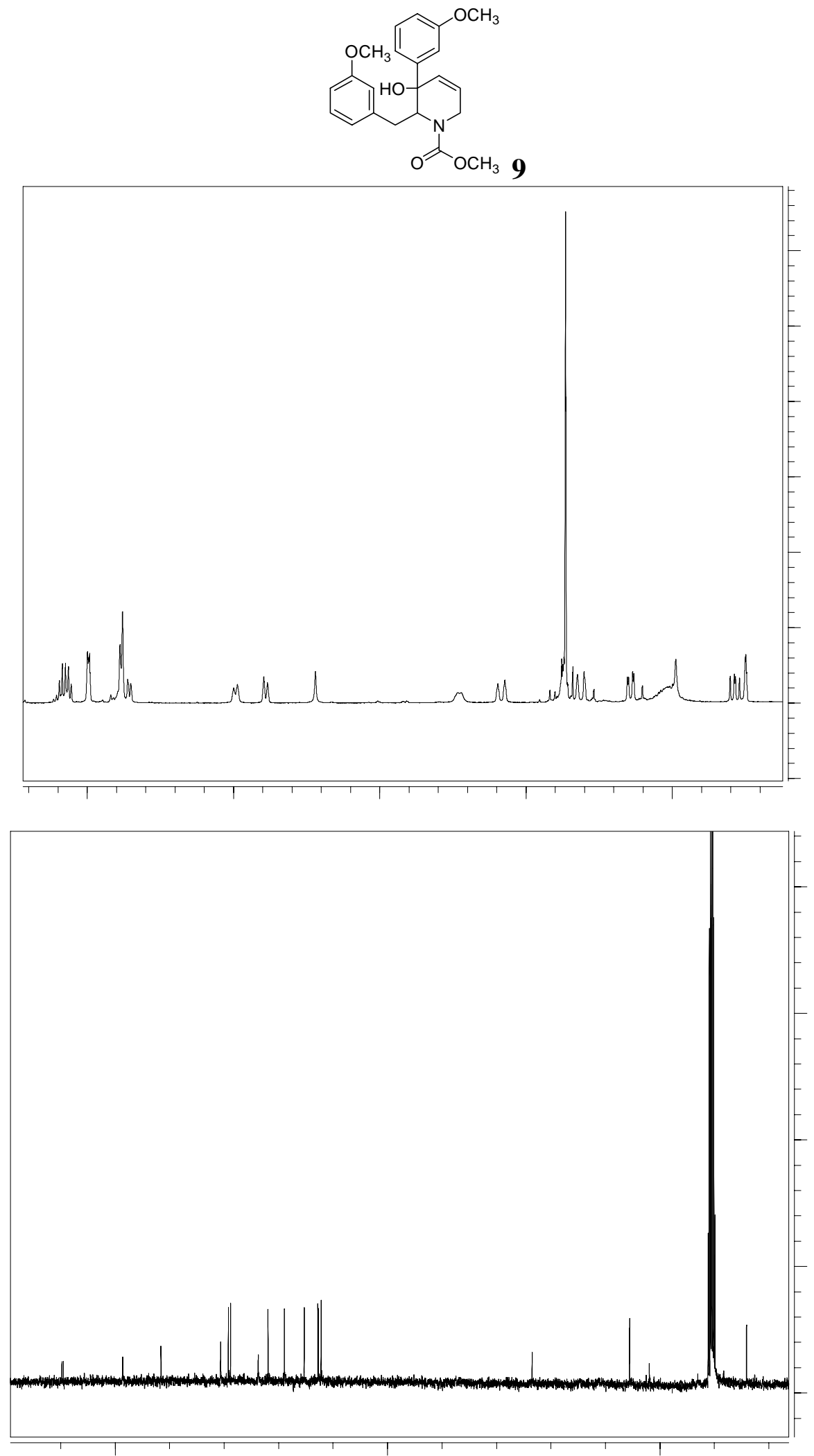

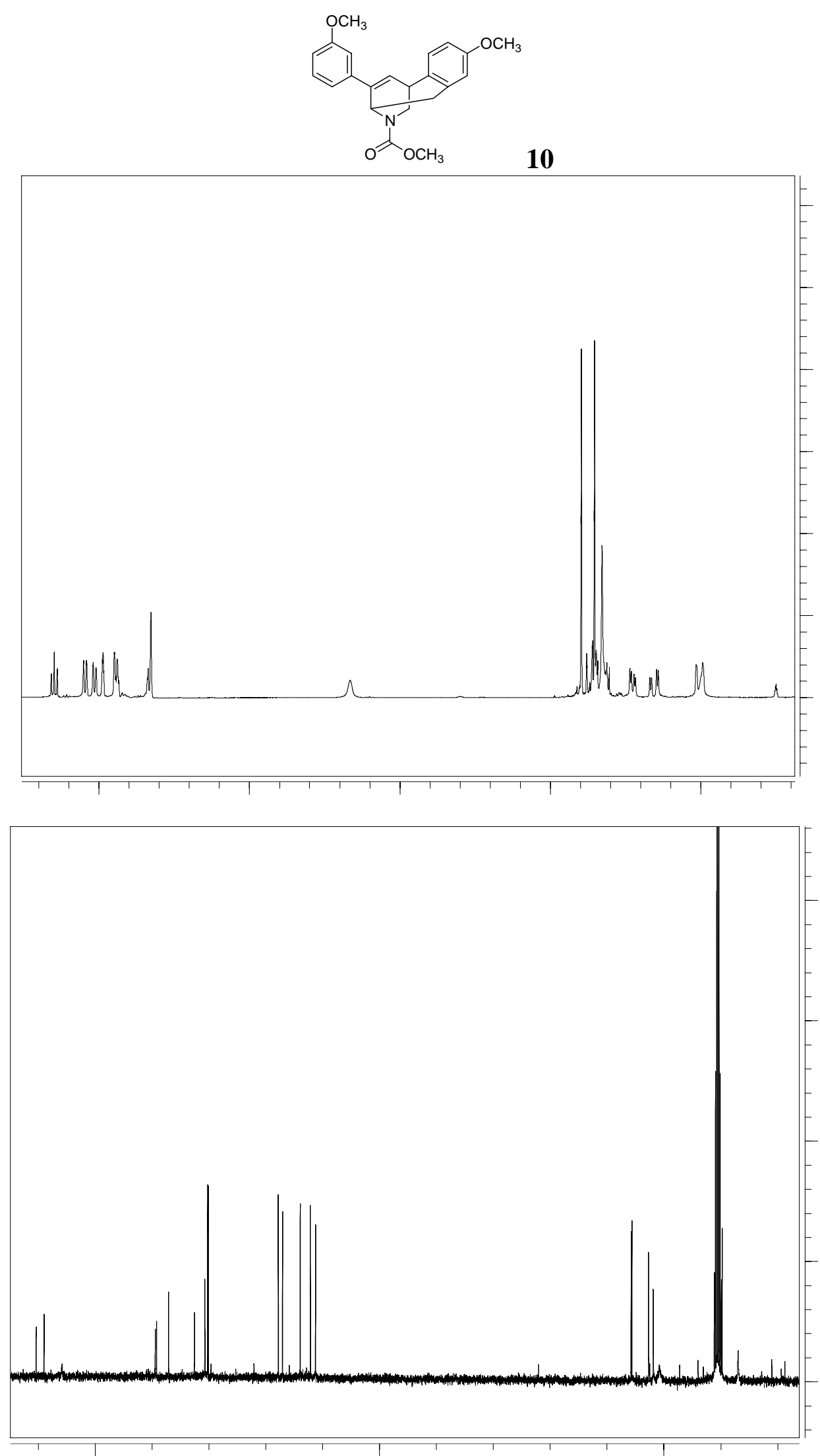


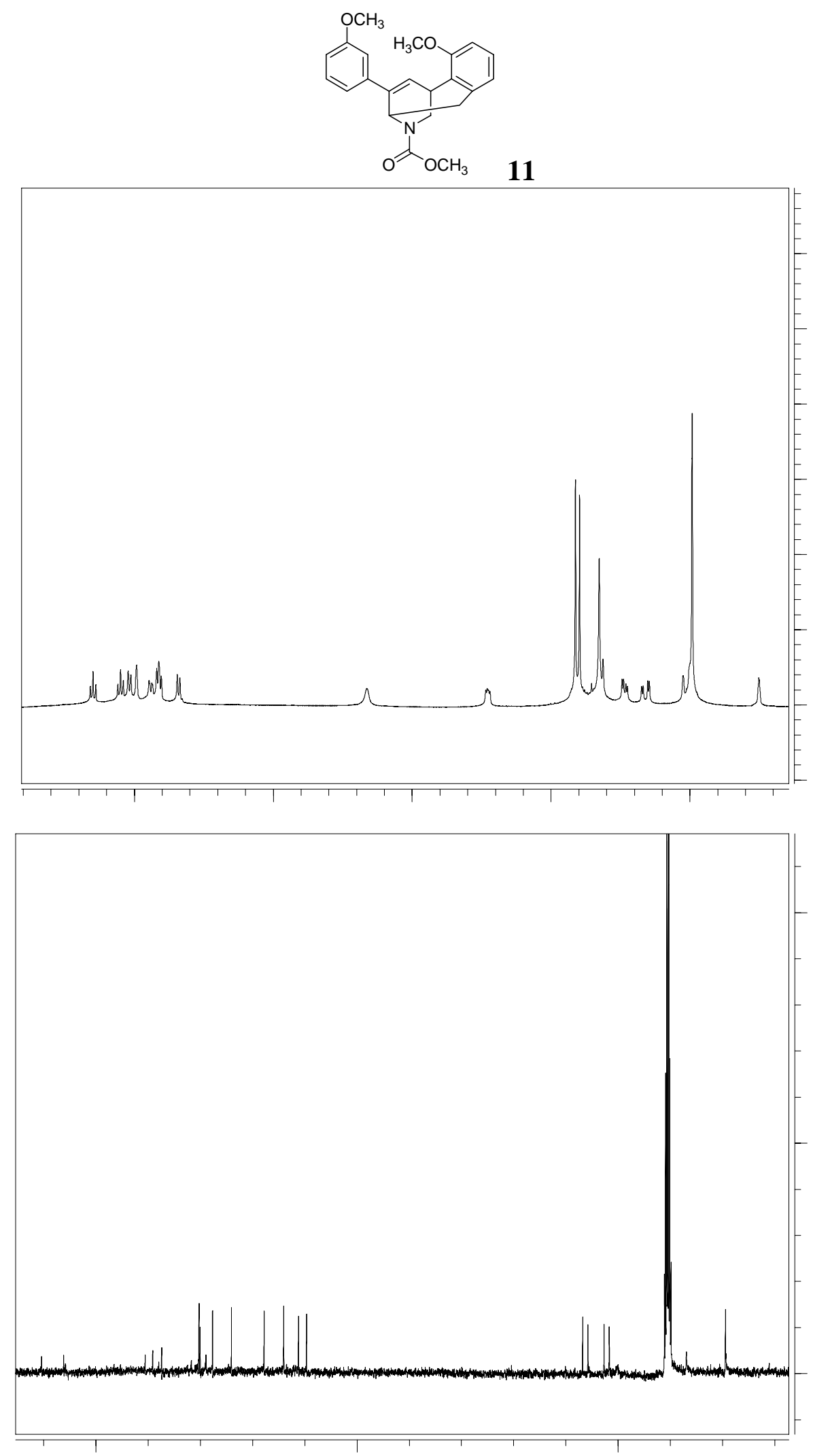



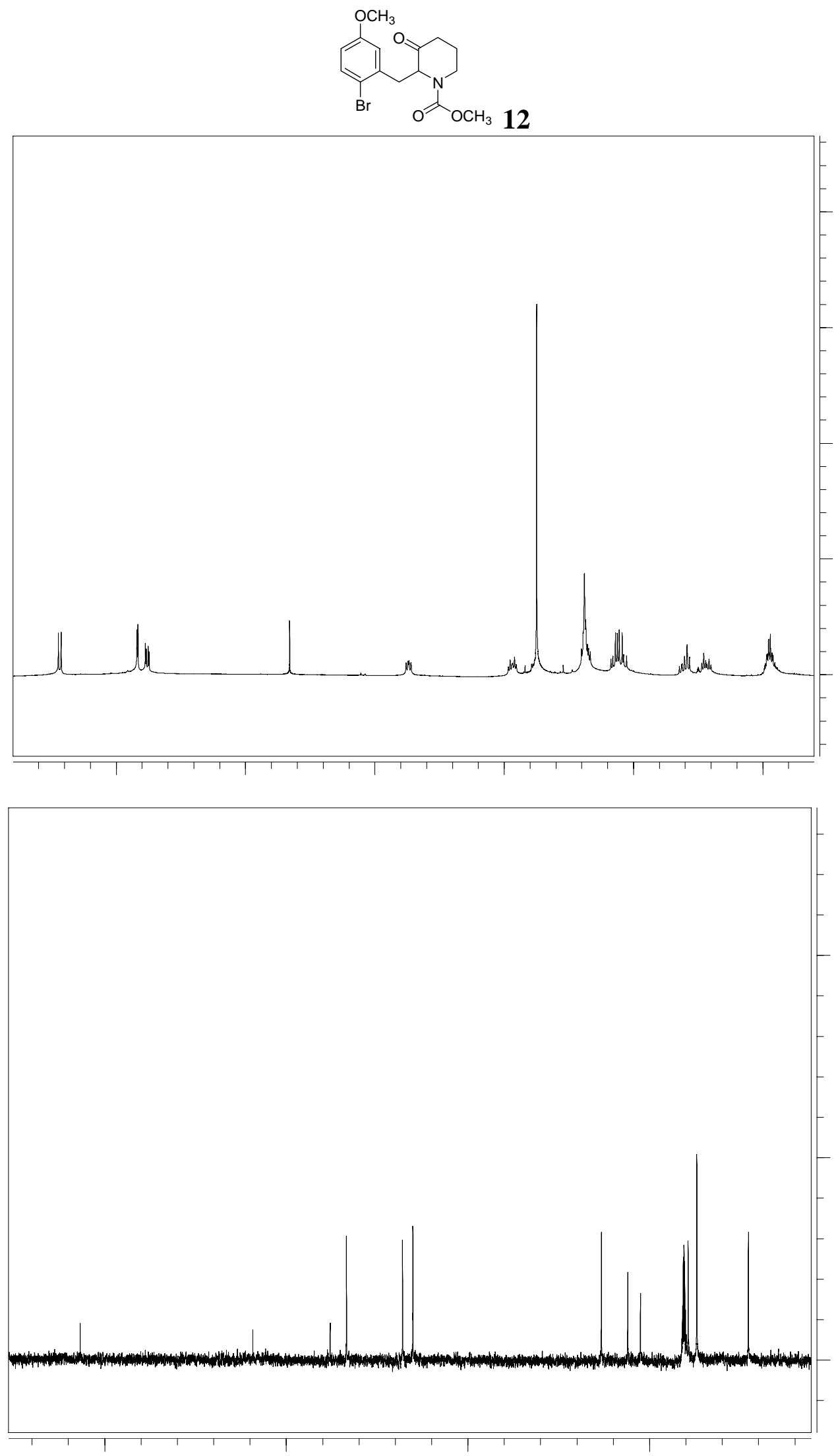

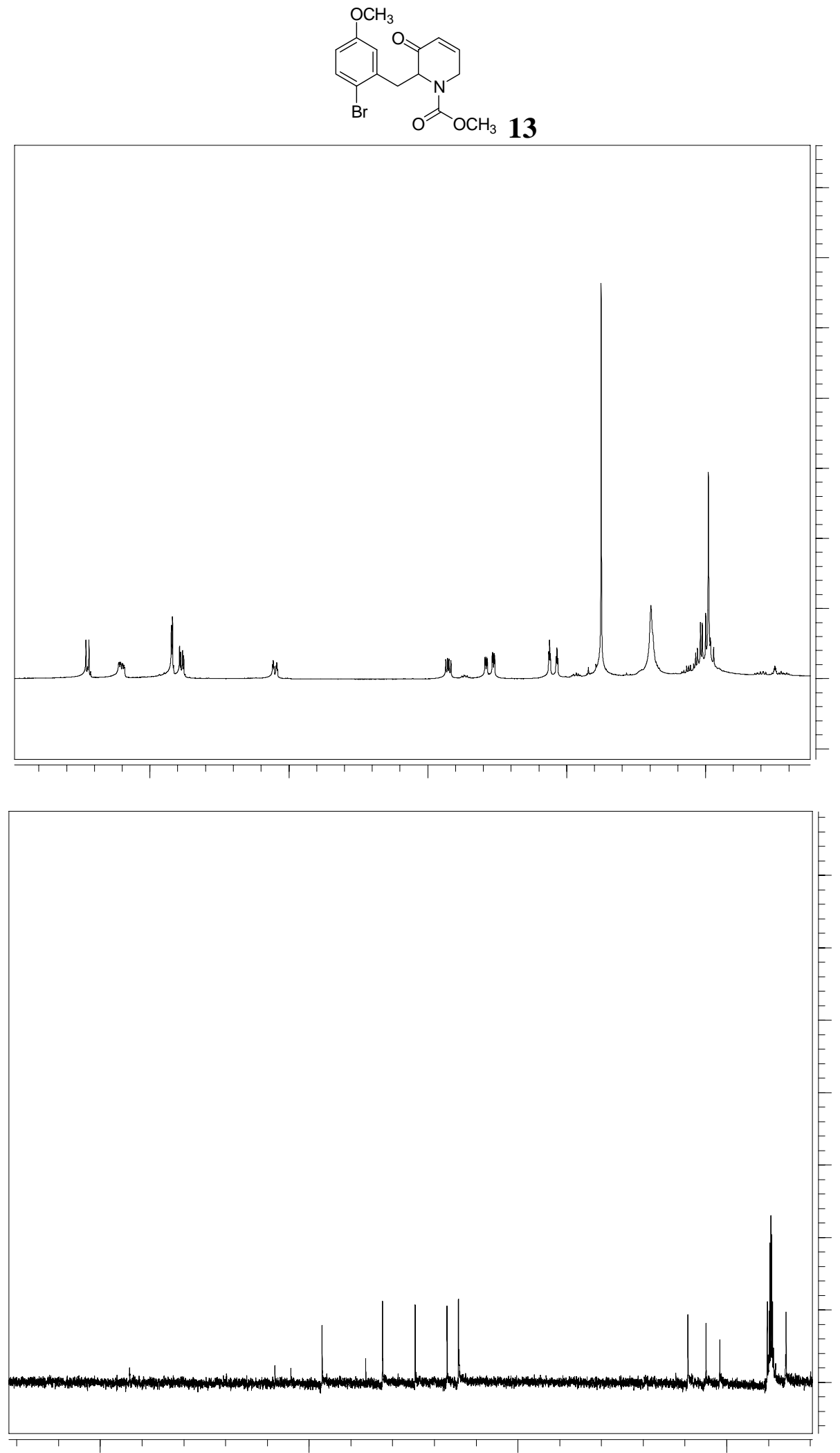

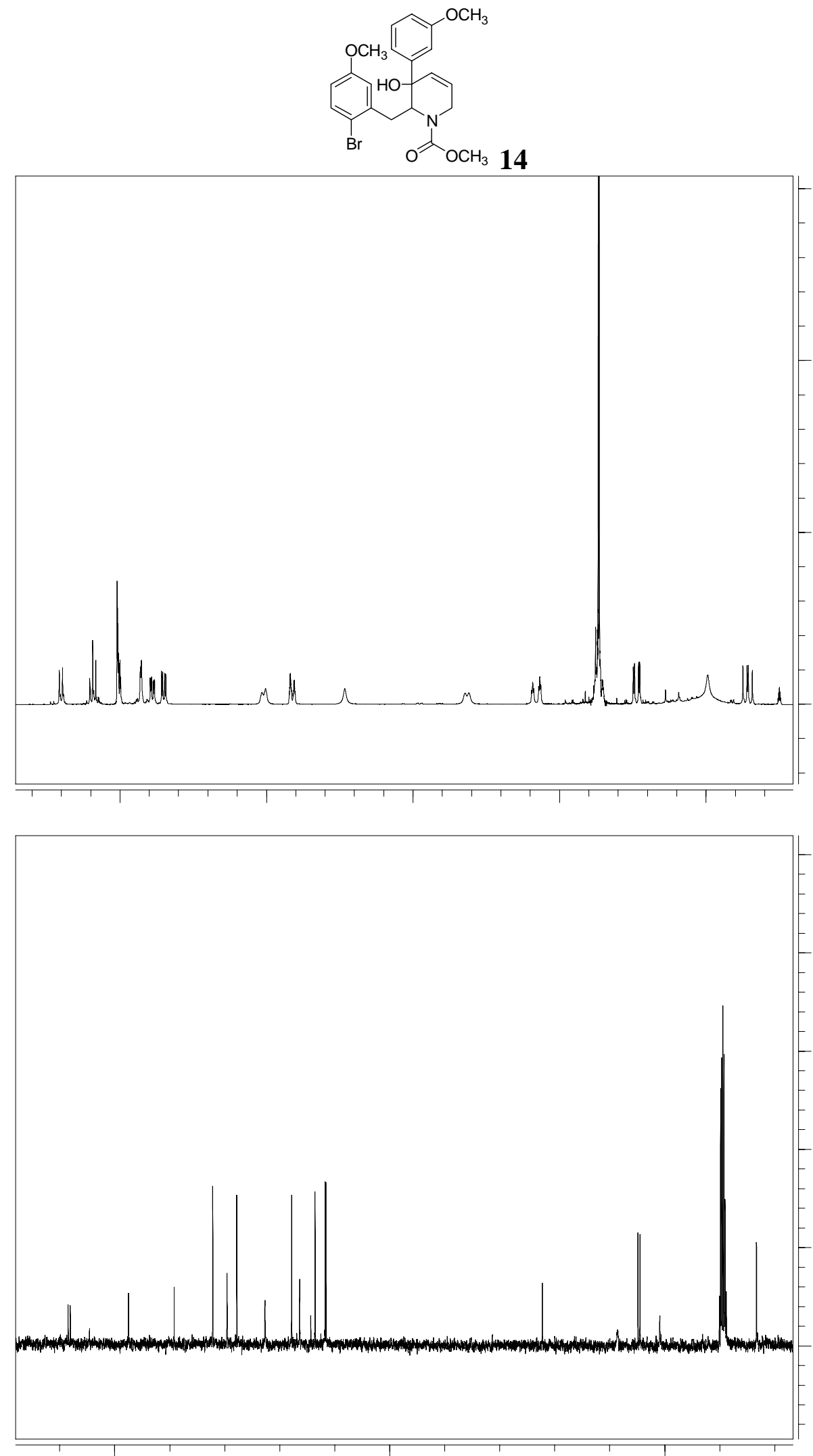

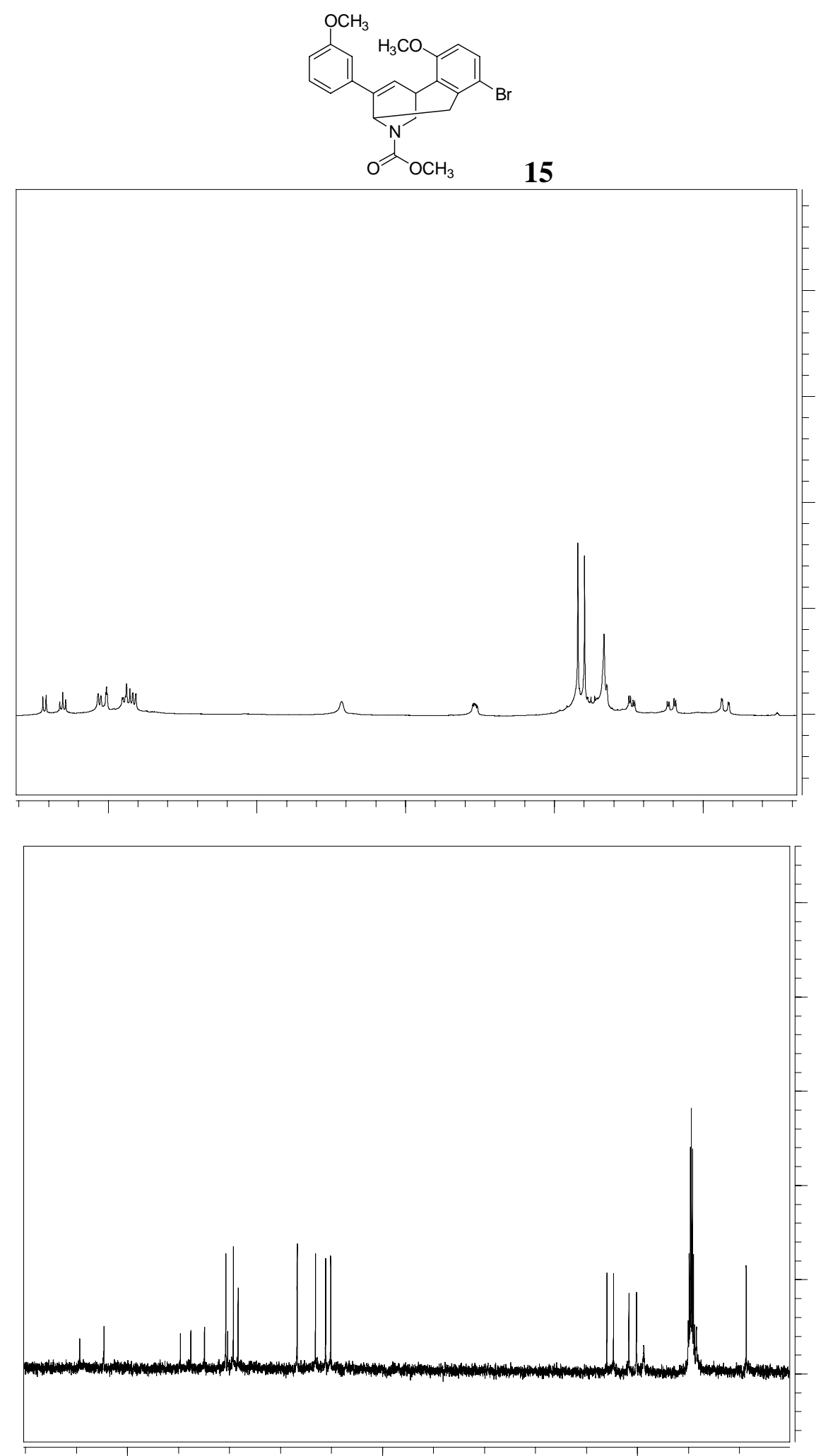

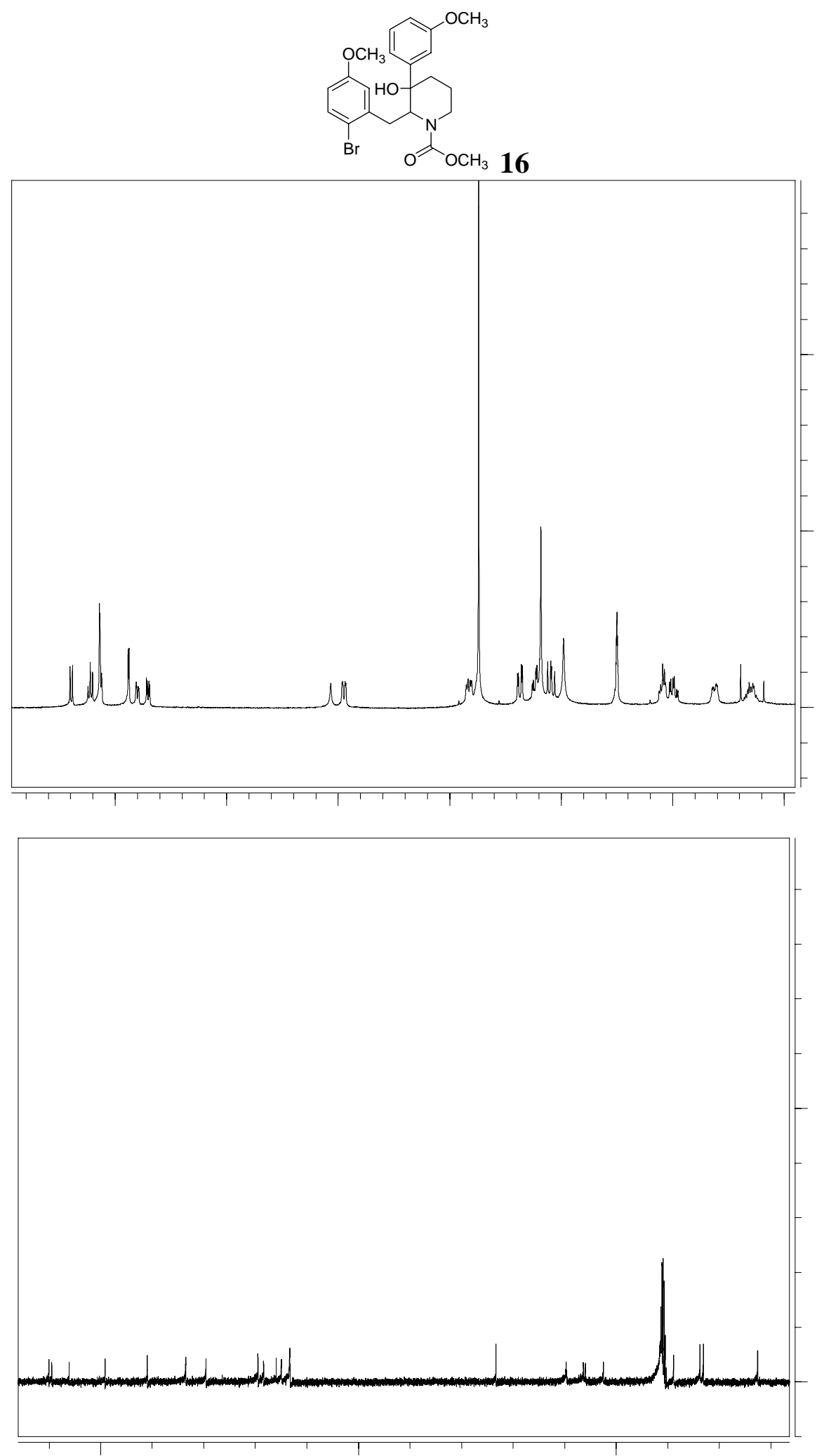

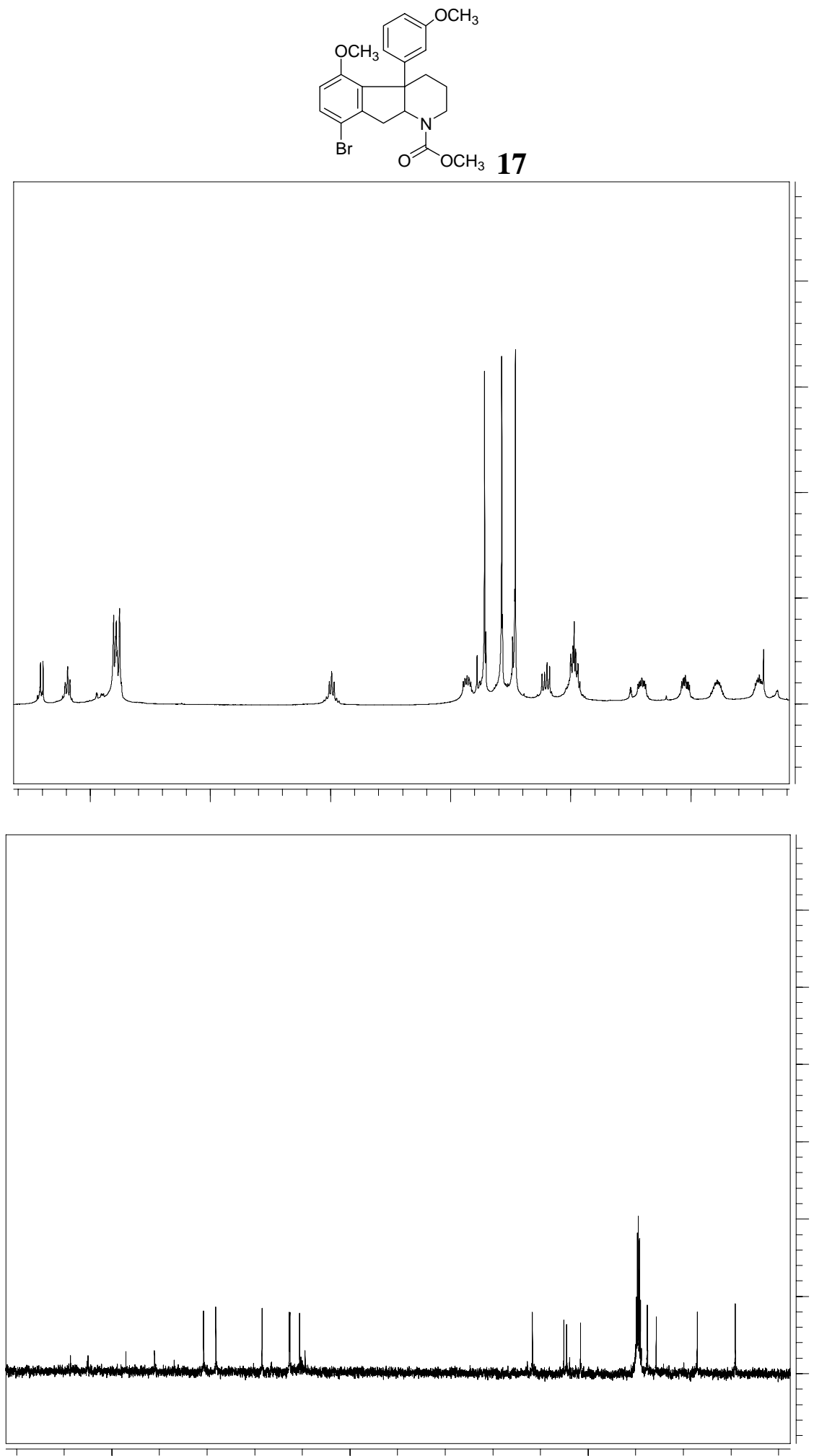

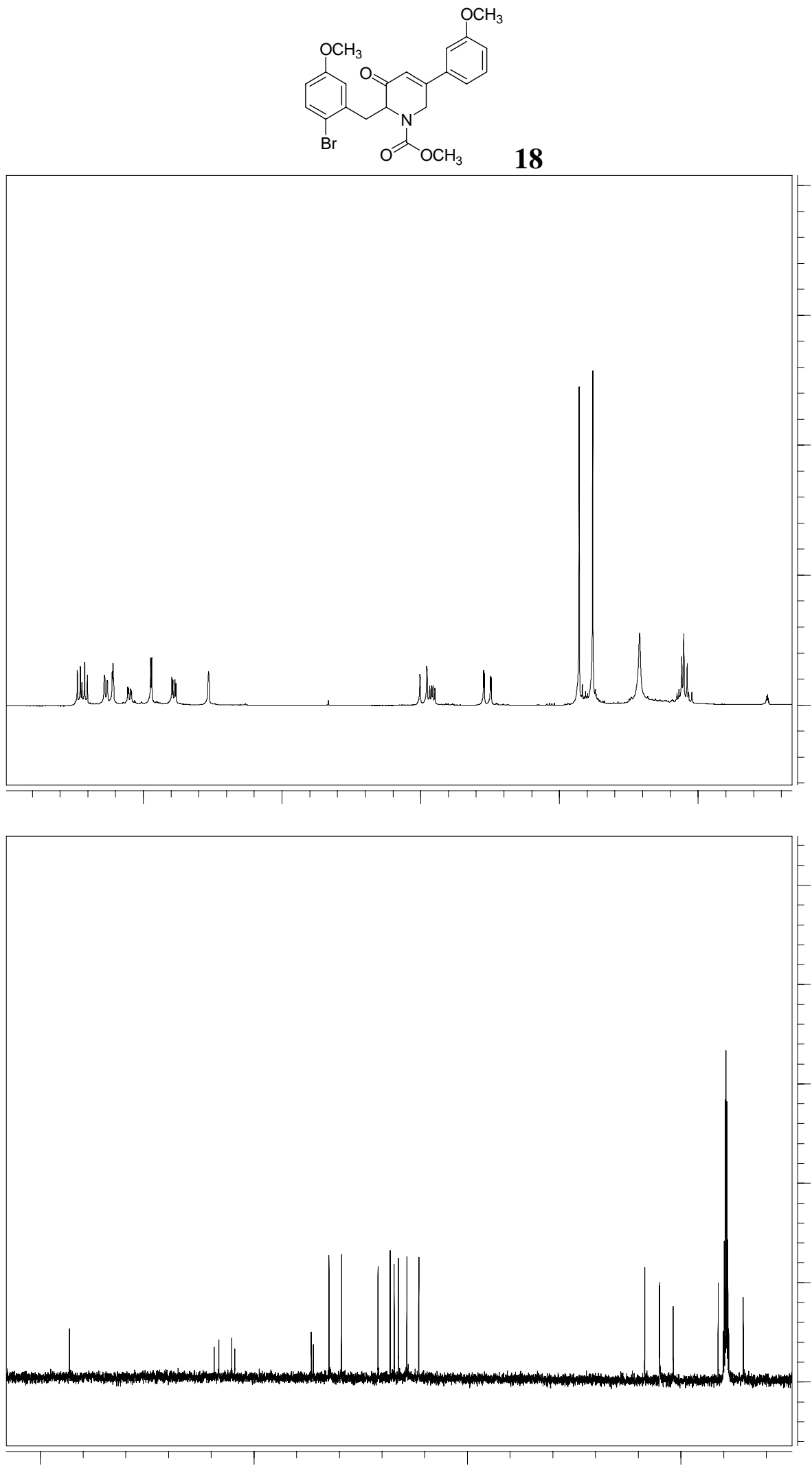


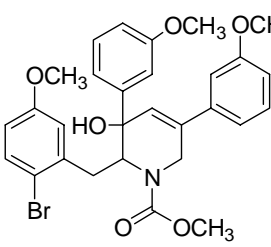

19
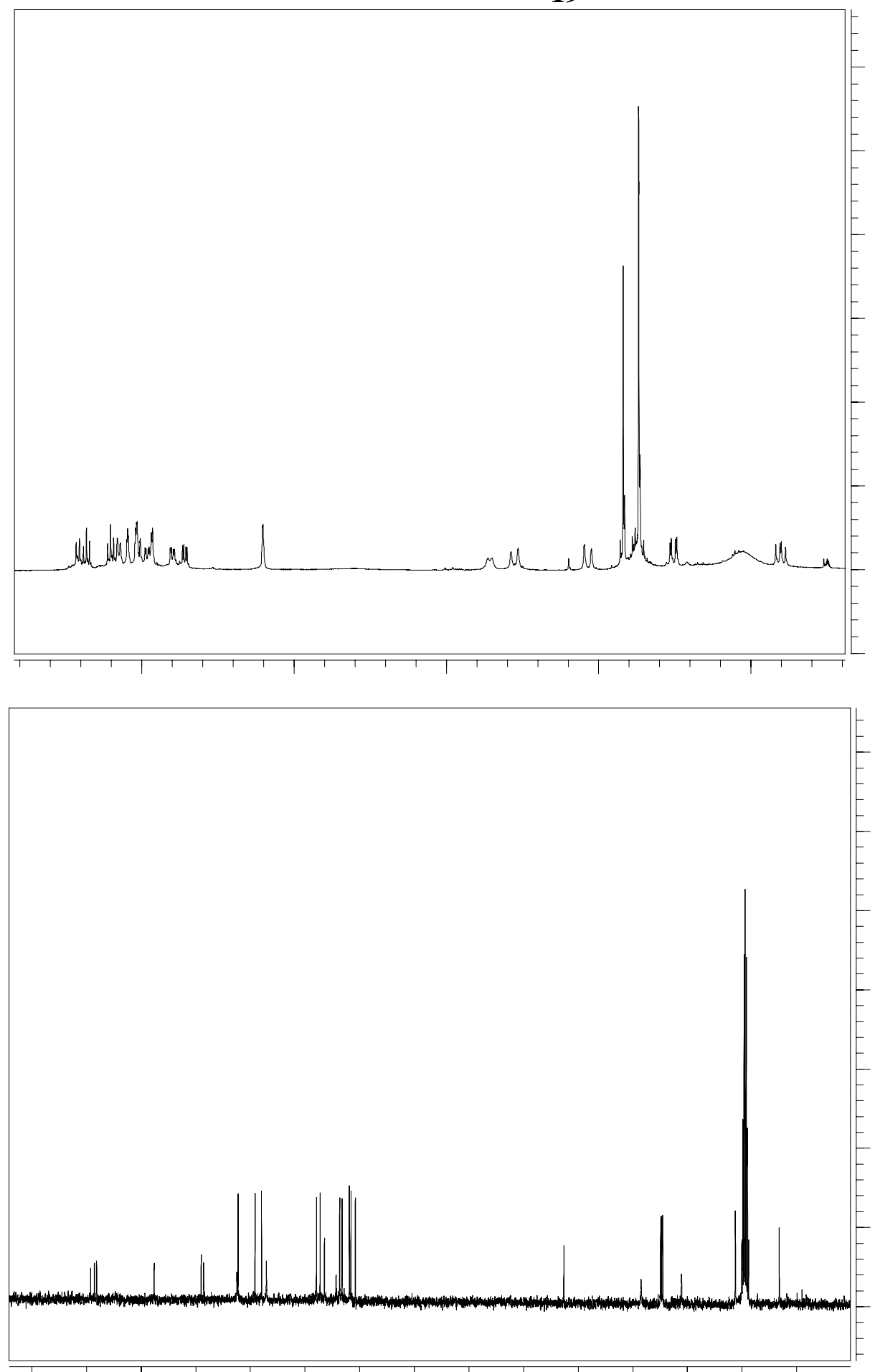

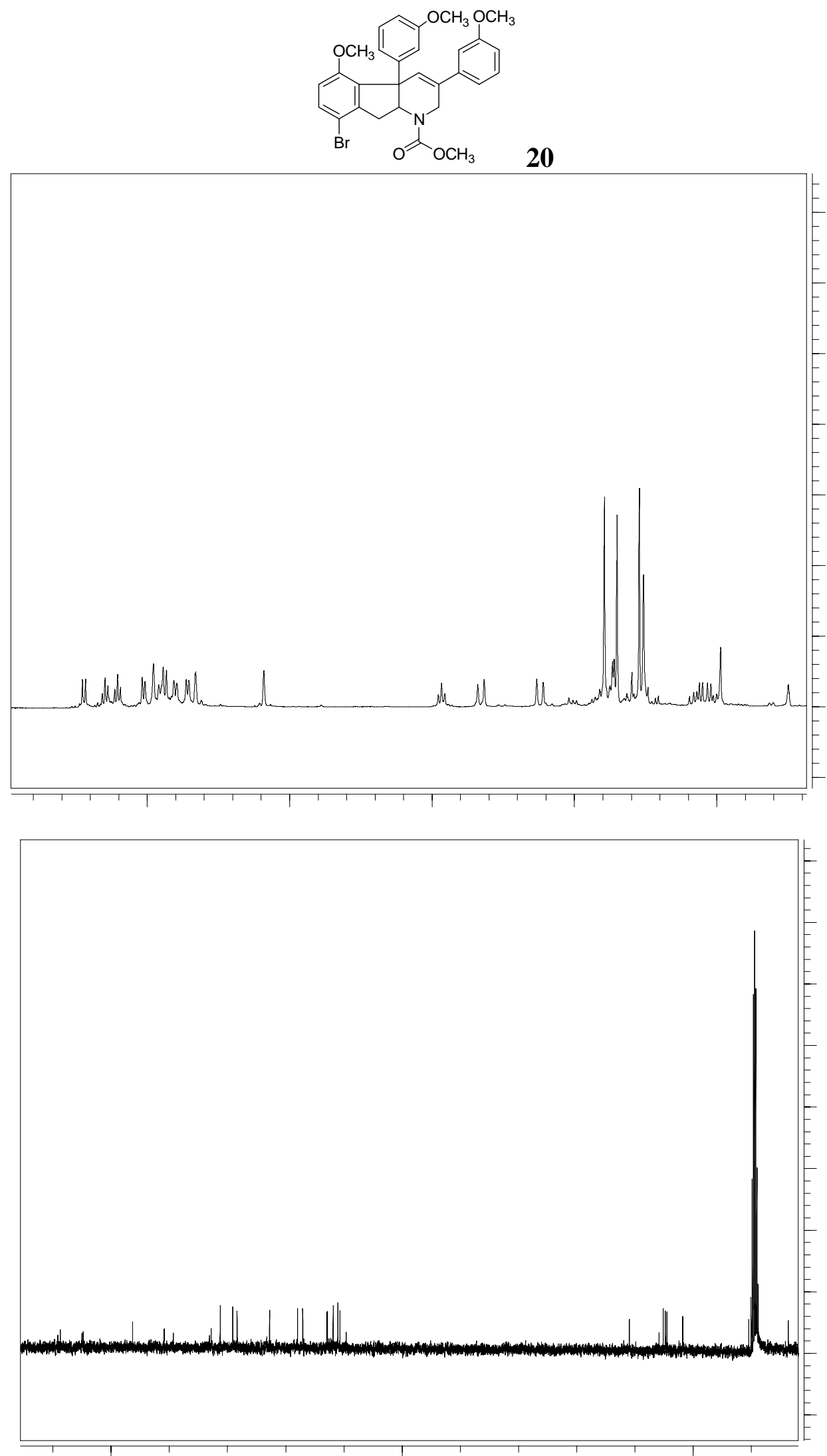